\title{
Tidal Response and Shape of Hot Jupiters
}

\author{
Sean M. Wahl ${ }^{1}$ (1), Daniel Thorngren ${ }^{2,3}$ (1) , Tiger Lu $^{4}$ (1) , and Burkhard Militzer ${ }^{1,5}$ (1) \\ ${ }^{1}$ Department of Earth and Planetary Science, University of California, Berkeley, CA 94720, USA \\ ${ }^{2}$ Department of Physics, University of California, Santa Cruz, USA \\ ${ }^{3}$ Institute for Research on Exoplanets, Université de Montréal, Canada \\ ${ }^{4}$ Astronomy Department, California Institute of Technology, Pasadena, CA 91125, USA \\ 5 Department of Astronomy, University of California, Berkeley, CA 94720, USA \\ Received 2021 April 3; revised 2021 July 21; accepted 2021 August 2; published 2021 November 5
}

\begin{abstract}
We study the response of hot Jupiters to a static tidal perturbation using the concentric MacLaurin spheroid method. For strongly irradiated planets, we first performed radiative transfer calculations to relate the planet's equilibrium temperature, $T_{\mathrm{eq}}$, to its interior entropy. We then determined the gravity harmonics, shape, moment of inertia, and static Love numbers for a range of two-layer interior models that assume a rocky core plus a homogeneous and isentropic envelope composed of hydrogen, helium, and heavier elements. We identify general trends and then study HAT-P-13b, the WASP planets 4b, 12b, 18b, 103b, and 121b, and Kepler-75b and CoRot$3 \mathrm{~b}$. We compute the Love numbers, $k_{n m}$, and transit radius correction, $\Delta R$, which we compare with predictions in the literature. We find that the Love number, $k_{22}$, of tidally locked giant planets cannot exceed a value of 0.6 , and that the high $T_{\text {eq }}$ consistent with strongly irradiated hot Jupiters tends to further lower $k_{22}$. While most tidally locked planets are well described by a linear regime response of $k_{22}=3 J_{2} / q_{0}$ (where $q_{0}$ is the rotation parameter of the gravitational potential), for extreme cases such as WASP-12b, WASP-103b, and WASP-121b, nonlinear effects can account for over $10 \%$ of the predicted $k_{22}$. The $k_{22}$ values larger than 0.6 , as they have been reported for planets WASP-4b and HAT-P13B, cannot result from a static tidal response without extremely rapid rotation and thus are inconsistent with their expected tidally locked state.
\end{abstract}

Unified Astronomy Thesaurus concepts: Exoplanets (498); Hot Jupiters (753); Exoplanet tides (497); Tides (1702); Tidal interaction (1699); Planetary interior (1248); Oblateness (1143); Tidal radius (1700); Gravitational fields

(667); Tidal distortion (1697)

\section{Introduction}

In this paper, we study the response of rotating giant exoplanets to tidal perturbations using the nonperturbative concentric MacLaurin spheroid (CMS) method (Hubbard 2013). The shape of a fluid planet results from a balance of tidal interactions with other celestial bodies and the planets' rotation with self-gravity from the planets' interior mass distribution. While the gas-giant planets in our solar system, Jupiter and Saturn, have had their interiors probed by precise spacecraft gravity measurements (Folkner et al. 2017; Iess et al. 2019), the tremendous distance to exoplanets necessitates more indirect means for studying their interiors. A class of exoplanets that is well suited for the study of tidal interactions is the hot Jupiters, whose relatively large masses and close-in orbits with their host star lead to much stronger tidal interactions than for any planet in our solar system. In addition, intense insolation allows their interiors to maintain much of their primordial heat, with equilibrium temperature $T_{\mathrm{eq}}>1000 \mathrm{~K}$ (Miller \& Fortney 2011), leading to their well-documented, inflated gaseous envelopes (Charbonneau et al. 2000; Henry et al. 2000; Guillot \& Showman 2002), which are even more prone to deformation by tidal interactions than colder planets.

The deformation of a planet can be characterized by the shape of its observable surface via radii along the principal axes, $a, b$ and $c$, or through normalized moments of its gravity field. For tidal interactions in particular, the first-order response is conventionally reported as the second-degree fluid Love number $k_{22}$ (Love 1909).

Batygin et al. (2009) identified a means of constraining $k_{22}$ for HAT-P-13b by considering its special orbital configuration with a highly eccentric outer companion planet. Buhler et al. (2016) applied this technique using observations of secondary eclipses to measure the eccentricity, $e$, and found $k_{22}=0.31_{-0.05}^{+0.08}$. Meanwhile, Hardy et al. (2017) used independent observations of HATP-13b secondary eclipses and inferred a much larger value of $k_{22}=0.81 \pm 0.10$. Ragozzine \& Wolf (2009) put forward another method for measuring $k_{22}$ by relating it to apsidal precession, which can manifest itself as transit timing variations (TTVs) between temporally separated transit observations. This method was applied to WASP-4b by Bouma et al. (2019), who estimated $k_{22}=1.20_{-0.26}^{+0.20}$ from TESS observations showing an offset in transit time with respect to predictions based on observations stretching back to 2007. Likewise, Csizmadia et al. (2019) estimated $k_{22}=0.62_{-0.19}^{+0.55}$ for WASP-18b using a similar technique but with the apsidal precession rate inferred from radial velocity (RV) variations instead. An initial suggestion of a detection of apsidal precision for WASP-12b was ruled out by Campo et al. (2011).

The shape of the observable surface of a sufficiently nonspherical planet can be inferred from transit light curves for both fast-rotating oblate planets (Seager \& Hui 2002) and tidally elongated prolate planets (Leconte et al. 2011; Burton et al. 2014). With the exception of special-case interactions with a secondary companion planet, most close-in hot Jupiters are expected to have evolved to a tidally locked state with negligible eccentricity (Lin $\&$ Gu 2004; Jackson et al. 2008). Because tidal locking limits the rotation rate to match the orbital period, the tidally induced prolateness is generally more pronounced than the rotational oblateness. For sufficiently close-in hot Jupiters, this can lead to a systematic underestimation of reported planetary radius and, by consequence, an overestimation of the bulk density. The effects of 
rotation and tidal perturbation were analyzed in detail by Leconte et al. (2011), who derived a predictive theory of how to correct the observed radii. We will compare our prediction of the radius correction of WASP-12b with their work. Correia (2014) formulated an analytical shape model with an assumed ellipsoidal shape and a tidal response following the Darwin-Radau equation and calculated $a, b$, and $c$ for a number of hot Jupiters, including WASP-4b, WASP-12b, WASP19b, and WASP-103b. Akinsanmi et al. (2019) applied Correia's shape model to predict the number of transits required to constrain the shape Love number $h_{2}$ of WASP-103b and WASP-121b for the TESS, PLATO, and JWST spacecraft. Similarly, Hellard et al. (2019, 2020) predicted the sensitivity of a number of spacecraft, including TESS, PLATO, and JWST, to measuring $k_{22}$ for WASP-121b.

There is extensive literature on the theory of calculating the shape of a liquid planet dating back over a century (H \& Jeans 1920), with pioneering calculations on giant planets presented in Gavrilov \& Zharkov (1977). The most commonly used method, known as the theory of figures (Zharkov \& Trubitsyn 1978), uses a perturbative approach to determine the planet's response to small deviations of the potential from spherical symmetry. Other works have extended the theory of figures to consider secondorder effects through higher-order perturbative theory (Zharkov 2004; Zharkov \& Gudkova 2010; Correia \& Rodríguez 2013). Padovan et al. (2018) adapted a related perturbative method using a matrix-propagator approach, more common in geophysical applications to exoplanets.

Hubbard (2013) introduced the CMS technique, a nonperturbative, iterative method for more precise calculations of selfconsistent shape and gravitational field. The CMS method was subsequently extended to three dimensions and applied to the cases of Jupiter and Saturn (Wahl et al. 2016, 2017a; Nettelmann 2019; Wahl et al. 2020). In this work, we apply the CMS method to hot Jupiter exoplanets for the first time. While more computationally expensive than the theory of figures, the CMS method correctly accounts for nonlinear effects that become relevant for extremely deformed planets, most notably effects on the order of the product of rotational and tidal perturbations (Wahl et al. 2017a). These nonlinear effects lead to a splitting of the static $k_{n m}$ with degree $m$ and an enhancement of $k_{22}$ that is significant for Jupiter and Saturn (Lainey et al. 2017, 2020; Durante et al. 2020; Wahl et al. 2020).

In addition to the numerical technique, models of shape and gravity also depend on the assumed interior structure and the hydrogen-helium equation of state (EOS). The relationship between tidal response and core mass is discussed in numerous works (e.g., Batygin et al. 2009; Ragozzine \& Wolf 2009), with more centrally concentrated density distributions leading to smaller values of $k_{22}$. Many studies of giant-planet interiors employ the semiempirical Saumon et al. (1995) EOS, while more recent studies have considered EOSs fit to ab initio molecular dynamics simulations of hydrogen-helium mixtures based on density functional theory molecular dynamics (DFT-MD; Militzer \& Hubbard 2013; Becker et al. 2015; Chabrier et al. 2019), while some theoretical works consider the simpler, more analytically tractable polytropic EOS (e.g., Leconte et al. 2011). The most pronounced difference between DFT-MD based EOSs and Saumon et al. (1995) occurs at pressures of $\sim 100 \mathrm{GPa}$; as hydrogen transitions from a molecular insulator to an atomic metal (Vorberger et al. 2010), DFT-MD predicts adiabatic temperature profiles that are are cooler and denser. Kramm et al. (2012) explored the possible interior structure of giant planet HAT-P13b

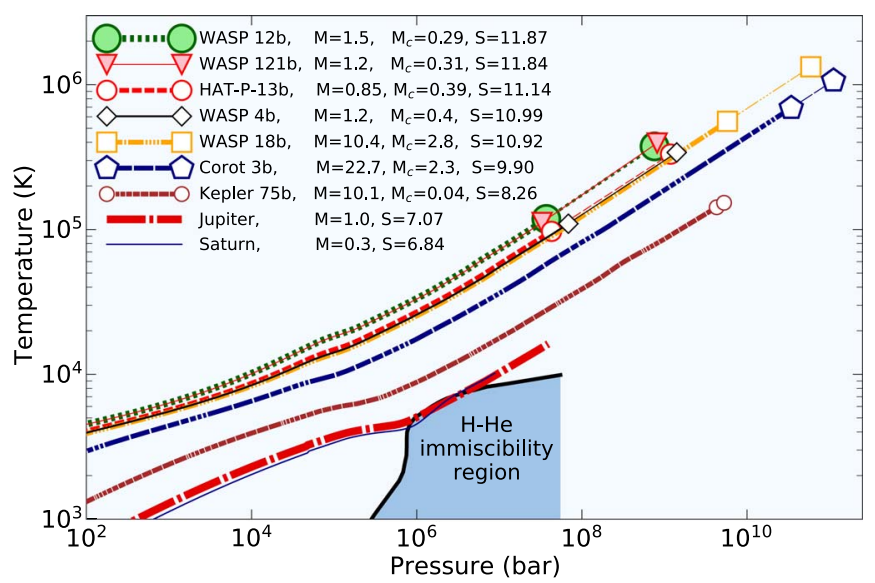

Figure 1. Pressure-temperature conditions in the deep interiors of selected planets. Masses of planets, $M$, and their cores, $M_{c}$, are given in Jupiter masses in the legend. The two symbols bracket conditions within the planet cores, from the core-envelope boundary (lower left) to the very center (upper right). The distance between the two symbols is primarily controlled by the ratio $M_{c} / M$. The entropy of the envelope is give in units of $k_{b} / \mathrm{el}$ in the legend. Adiabats with $S \leqslant 7.2 k_{b} / \mathrm{el}$ intersect the hydrogen-helium immiscibility region (Morales et al. 2010).

using interior models with the Saumon et al. (1995) EOS and the theory of figures to calculate the Love number, $k_{22}$. Becker et al. (2018) calculated Love numbers using a DFT-MD EOS and the theory of figures for two giant exoplanets, Kepler-75b (formerly KOI-889b) and CoRoT-3b. A similar study of planets in the superEarth exoplanet regime was carried out by Kellermann et al. (2018).

\section{Methods}

We first solve the equations of hydrostatic equilibrium for a nonrotating planet as described in Seager et al. (2007). For the EOS of hydrogen-helium mixtures, we adopt the results from Militzer \& Hubbard (2013), who employed density functional theory molecular dynamics (DFT-MD) simulations to derive an EOS table with absolute entropies at pressures higher than $\sim 5 \mathrm{GPa}$. At lower pressures, we use the Saumon-Chabrier EOS that was derived with semianalytical methods (Saumon et al. 1995). Heavier elements are incorporated into the $\mathrm{H}-\mathrm{He}$ mixture by following the approach in Hubbard \& Militzer (2016). For the core, we adopted a terrestrial iron-rock ratio of 0.325 . For simplicity, we assumed that both components are homogeneously mixed. The silicates are described as in Seager et al. (2007). For iron, we employed results from DFT-MD simulations (Wilson \& Militzer 2014).

For all calculations, we assume a protosolar value for the helium mass fraction, $Y$, of the envelope by setting $Y /(1-Z)=$ 0.27774 (Lodders 2010). The fraction of heavy elements, $Z$, and the entropy, $S$, are input parameters of our simulations, which govern the density structure of the hydrogen-helium envelope. We provide entropy values in units of Boltzmann constant per electron $\left(k_{\mathrm{B}} / \mathrm{el}\right)$, which is referenced to an atomic $\mathrm{H}:$ He ratio of 110:9 from Militzer \& Hubbard (2013). In these units, helium rain is predicted to start at $S=7.2 k_{\mathrm{B}} / \mathrm{el}$, the maximum entropy for which the interior adiabat intersects the pressure-temperature region in Figure 1, in which hydrogen and helium are predicted to become immiscible (Morales et al. 2010) because hydrogen transitions from an insulating, molecular state to an atomic, metallic fluid (Vorberger et al. 2010) while helium remains in an insulating state. In this work, however, we are primarily concerned with hot Jupiters that we 
assume to have homogeneously mixed envelopes with entropies $S \geqslant 7.2 k_{\mathrm{B}} / \mathrm{el}$.

In addition to the $S$ and $Z$ values for the envelope, we adopt values for the masses of the core and envelope (see Table 1). We integrate the equations of hydrostatic equilibrium starting from a central pressure, $P_{c}$, to the outer pressure boundary, set to 1 bar, where we assume that the envelope becomes transparent. We iterate over different $P_{c}$ values to match the total mass of the planet. In cases for which we have a radius measurement, we iterate over the core mass or envelope $Z$ to match the planet's mass and radius simultaneously.

The planet mass, $M$, and volumetric average radius, $a_{0}$, from an initial calculation of the planet in a not-rotating state, define the planetary units of mass and length for all of the following CMS calculations for rotating and tidally perturbed planets. In absence of a tidal perturbation, we express the gravitational potential of an axisymmetric rotating planet,

$$
V(r, \mu)=\frac{G M}{r}\left[1-\sum_{n=1}^{\infty}\left(\frac{a_{0}}{r}\right)^{2 n} J_{2 n} P_{2 n}(\mu)\right],
$$

in terms of the gravity harmonics,

$$
J_{n}=-\frac{2 \pi}{M a_{0}^{n}} \int_{-1}^{+1} d \mu \int_{0}^{r_{\max }(\mu)} d r r^{n+2} P_{n}(\mu) \rho(r, \mu) .
$$

Here $r_{\max }$ defines the outer surface of the planet as a function of $\mu=\cos (\theta)$, with $\theta$ being the polar angle; $P_{n}$ are the Legendre polynomials; and $G$ is the gravitational constant. We also define two rotational parameters,

$$
q_{0}=\frac{\omega^{2} a_{0}^{3}}{G M} \text { and } q_{\mathrm{e}}=\frac{\omega^{2} a_{\mathrm{e}}^{3}}{G M},
$$

where $\omega$ is the angular frequency of the planet's assumed solidbody rotation, and $q_{\mathrm{e}}$ is often invoked in the literature when specific planets are discussed for which the equatorial radius, $a_{\mathrm{e}}$, is known while $a_{0}$ is not. For the purposes of this paper, $q_{0}$ is more convenient because it does not depend on $\omega$ or the equatorial radius, which will only become known once the CMS calculation has converged.

The CMS technique (Hubbard 2013) is a nonperturbative method for deriving the shape and interior structure of rotating planets in hydrostatic equilibrium. Typically, one keeps the equatorial radius constant and adjusts the core mass or $Z$ of the envelop to match the observed mass of the planet (e.g., Hubbard \& Militzer 2016). This approach does not serve our needs because, for different rotation rates, we wish to study what shape is assumed by a planet of given core and envelope masses. The equatorial radii of the planet (and core) are results, not input parameters, of such a calculation. Hubbard (2013) introduced a grid of $\lambda$ points, normalized radii from the planet's center to the equator that anchor the equatorial points of all of the equipotential surfaces as the CMS converges toward a self-consistent hydrostatic solution. Here we work with two $\lambda$ grids, one for the core and one for the envelope. Following Militzer et al. (2019), for the $j$ th spheroid surface, $\lambda_{j}$ is chosen so that a logarithmic grid in density emerges $\left(\rho\left(\lambda_{j}\right) / \rho\left(\lambda_{j+1}\right)=\right.$ const $)$. We determine the $\rho(\lambda)$ relation from a nonrotating planet calculation, which we then employ for the subsequent calculations with rotation and tides. The density variations throughout the core and envelope determine how many grid points must be invested into representing each region accurately. We used 1025 layers in our reported CMS calculations, but even with 129 layers, one already obtains good results. For example, for a WASP-12b model with a $0.29 M_{\mathrm{J}}$ core and 129 layers, we calculate the Love number $k_{22}=0.16258843$ and transit radius correction $\Delta R=0.034046$. With 1025 layers, we derived $k_{22}=0.16483250$ and $\Delta R=0.031489$, which are both fairly similar. As we will demonstrate, these deviations are small compared to those resulting from changed model assumptions and planet parameters.

In order to match the core and envelope masses in our CMS calculations of rotating planets, we rescale the $\lambda$ grids of the core and envelope separately as the CMS method converges to a hydrostatic solution. This poses no technical challenges unless the planets are rotating extremely fast $\left(q_{0} \gtrsim 0.3\right)$.

Once this axisymmetric CMS calculation has converged, we study the planet's shape, compute the gravity harmonics $J_{n}$, and derive the moment of inertia, $C /\left(M a_{0}^{2}\right)$. The hydrostatic structure of axisymmetric CMS calculation also serves as input for a 3D CMS calculation that studies the static tidal response to an external perturber, which could be the planet's host star, a satellite, or a companion planet.

The CMS technique was extended to three dimensions by Wahl et al. (2017a). In this version, a third potential term, the gravitational potential from a perturbing mass, $m_{S}$, at a distance, $\boldsymbol{R}$, from the planet's center of mass,

$$
W(\boldsymbol{r}, \boldsymbol{R})=\frac{G m_{S}}{|\boldsymbol{R}-\boldsymbol{r}|},
$$

is added to Equation (1), and equipotential surfaces for the combined potential are evaluated on a 3D grid $\boldsymbol{r}(r, \mu, \phi)$. Wahl et al. (2020) updated the 3D CMS method by modifying $W$ by subtracting out a linear term determined by an average force,

$$
\tilde{W}(\boldsymbol{r}, \boldsymbol{R})=W(\boldsymbol{r}, \boldsymbol{R})-\langle\mathbf{F}\rangle \cdot \boldsymbol{r} .
$$

This enforces the constraint that the planet's center of mass remains at a specified distance from the perturber. This procedure avoids an issue where the precision of the converged solution is limited by a small shift in the center of mass that must be removed from each iteration (Wahl et al. 2016, 2017a).

As with the rotational parameter, we can define two tidal parameters,

$$
q_{\mathrm{tid}, 0}=-3 \frac{m_{S} a_{0}^{3}}{M R^{3}} \text { and } q_{\mathrm{tid}, \mathrm{e}}=-3 \frac{m_{S} a_{\mathrm{e}}^{3}}{M R^{3}},
$$

where $m_{S}$ is the mass of the perturber (the stellar mass for the purposes of this study), and $R$ is the distance to the perturber, in our case, the orbital distance. We once again elect to use $q_{\mathrm{tid}, 0}$ for convenience and refer to it simply as $q_{\text {tid }}$ for the remainder of the paper. The third and final governing parameter for the tidal calculation is simply the ratio of the planet's radius to the orbital radius, $a_{0} / R$. In our own solar system, the rapid rotation of Jupiter and Saturn place them in the regime where $q_{0} \gg\left|q_{\text {tid }}\right|$, even for their strongest perturbers, Io and Tethys, respectively. In contrast, tidally locked hot Jupiters typically have values of $q_{\text {tid }}$ of a similar or greater magnitude than $q_{0}$.

As in the axisymmetric case, the converged equipotential surfaces define a density structure from which gravitational field strength can be integrated, now in terms of the tesseral gravity moments $C_{n m}$ and $S_{n m}$ (Wahl et al. 2017b). For simplicity, we assume that the perturber is in the planet's equatorial plane with $\mu=\cos \theta=0$. For an exoplanet-star system, this corresponds to 
Table 1

Parameters of Our Two-layer Interior Models

\begin{tabular}{ll}
\hline \hline $\begin{array}{l}\text { Planet mass } M\left[M_{\mathrm{J}}\right] \\
\text { Core mass } M_{c}\left[M_{\oplus}\right]\end{array}$ & $\begin{array}{l}\text { Input parameter. } \\
\text { Input parameter. Sets the envelope mass to } M-M_{c} . \text { Core shape and radius are derived. }\end{array}$ \\
\hline Entropy of envelope $S\left[k_{B} / \mathrm{el}\right]$ & $\begin{array}{c}\text { Input parameter chosen between } 7.2 \text { and 12.0. Sets the temperature-pressure profile of the envelope in Figure 1. } \\
\text { Derived from } T_{\text {eq }} .\end{array}$ \\
\hline Mass fraction of heavy elements in envelope $Z$ & $\begin{array}{l}\text { Input parameter that also sets the mass fractions of hydrogen, } X=1-Y-Z \text {, and helium, } Y=0.27774 \times(1-Z), \\
\text { because we assume a protosolar helium abundance (Lodders 2010). }\end{array}$ \\
\hline Planet radius $a_{0}\left[R_{\mathrm{J}}\right]$ & Derived in calculations of nonrotating planet. Alternatively, $Z$ or $M_{c}$ can be adjusted to match a certain radius.
\end{tabular}

a planet with zero obliquity. While this is not a good approximation for the exoplanet population as a whole, it is likely to be the case for many close-in hot Jupiters (Lin \& Gu 2004; Jackson et al. 2008). We further simplify the geometry by defining the coordinate system such that the perturber is at $\phi=0$, which, by symmetry, requires $S_{n m}=0$. The tidal Love number can then be expressed as (Gavrilov \& Zharkov 1977; Zharkov \& Trubitsyn 1978)

$$
k_{n m}=-\frac{2}{3} \frac{(n+m) !}{(n-m) !} \frac{C_{n m}}{P_{n}^{m}(0) q_{\mathrm{tid}, 0}}\left(\frac{a_{0}}{R}\right)^{2-n},
$$

where $P_{n}^{m}(0)$ is the associated Legendre polynomial evaluated at $\mu=0$. For a distant perturber, the $m=2$ moment dominates the expansion, but higher-order moments become more significant as $a_{0} / R$ increases. We note that the Jupiter and Saturn $k_{22}$ reported later for comparison defined $k_{n m}$ with $q_{\text {tid,e }}$ instead of $q_{\mathrm{tid}, 0}$ (Wahl et al. 2017a, 2020).

In the absence of rotation, $k_{n m}$ is degenerate with respect to $m$. By contrast, rapidly rotating planets, such as Jupiter and Saturn, are predicted to have significant splitting of Love numbers of the same order $n$ (Wahl et al. 2016, 2017a, 2020). The most readily observable manifestation of this is an enhancement of $k_{22}$ compared to a nonrotating analog planet, which is evident for observations of both Jupiter (Durante et al. 2020) and Saturn (Lainey et al. 2017, 2020).

\section{Results and Discussion}

\subsection{General Trends}

In Figure 2, we compare the gravity harmonics of four Saturn-mass planets with $10 M_{\oplus}$ cores as a function of the rotational parameter, $q_{0}$. By definition, all curves start from $J_{0}=-1$. All cases decay exponentially with increasing degree, $n$, but their decay rates vary with the magnitude of $q_{0}$. For slowly rotating planets, $J_{n}$ decays most rapidly, which is consistent with the fact that all $J_{n \geqslant 2}$ are zero for a nonrotating planet. With increasing degree, the weight functions of the gravity coefficients become more sharply peaked near the surface (Militzer et al. 2016). This means that $J_{n}$ decays more rapidly for a hot, puffy planet $\left(S=11 k_{b} / \mathrm{el}\right)$ that has less mass near the surface. It also implies that $J_{n}$ decays more slowly for fast-rotating planets, for which the centrifugal force shifts more mass toward the equator.

In Figure 3, we compare various properties of a cold and hot Saturn-mass planet $\left(S=7.2\right.$ and $\left.11.0 k_{b} / \mathrm{el}\right)$ and a cold Jupitermass planet $\left(S=7.2 k_{b} / \mathrm{el}\right)$, each with a $10 M_{\oplus}$ core. For moderate values of $q_{0}$, we find that the gravity harmonics, $J_{2}$, $J_{4}$, and $J_{6}$, scale approximately as $q_{0}, q_{0}^{2}$, and $q_{0}^{3}$, respectively, because with increasing $q_{0}$, additional mass is shifted toward

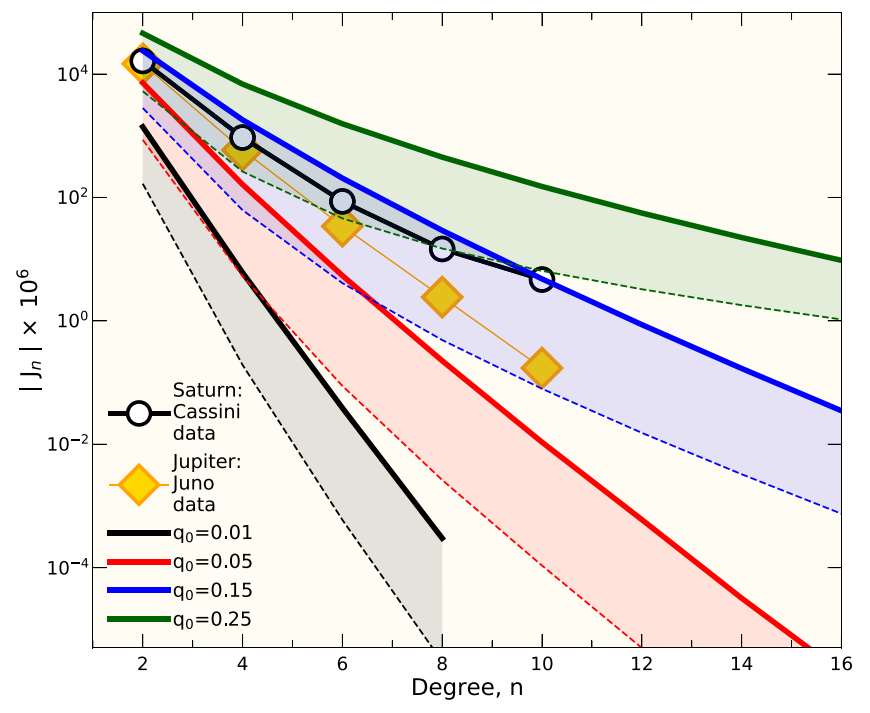

Figure 2. Decay of the gravity harmonics with increasing degree, $n$, for Saturnmass planets that rotate at different rates. The thick solid lines and thin dashed lines correspond to cold $\left(S=7.2 k_{b} / \mathrm{el}\right)$ and hot $\left(S=11.0 k_{b} / \mathrm{el}\right)$ planets, respectively. Spacecraft measurements of Jupiter and Saturn have been included for comparison. The unexpectedly large values of Saturn's $J_{6}, J_{8}$, and $J_{10}$ have been attributed to differential rotation (Iess et al. 2019), which we do not include in our exoplanet models because there are no observations to constrain the winds on these planets.

the equator. For all three planets, we find a sizable increase in the equatorial radius for large $q_{0}$. However, the polar radius only shrinks significantly for the two colder planets. The hot Saturn-mass planet is so inflated $\left(a_{0}=2.6 R_{\mathrm{J}}\right)$ and the centrifugal force as large for high $q_{0}$ that the polar radius hardly shrinks as the equatorial radius increases.

In the bottom panel of Figure 3, we plot the moment of inertia. For the hot, puffy Saturn-mass planet, it hardly changes over the $q_{0}$ interval from zero to 0.25 . For the two colder planets, we see a modest increase in the moment of inertia for $q_{0}>0.1$ as the centrifugal force distributes more mass away from the axis of rotation.

The Darwin-Radau relation gives an approximate expression for the moment of inertia, $C$, of slowly rotating planets (see discussion in Zharkov \& Trubitsyn 1978),

$$
\begin{gathered}
\frac{C}{M a_{\mathrm{e}}^{2}}=\frac{2}{3}\left(1-\frac{2}{5} \sqrt{x}\right), \\
x_{1}=1+\eta \text { with } \eta=\frac{5}{2} \frac{q_{\mathrm{e}}}{f} \text { and } f=\frac{a_{\mathrm{e}}-c}{a_{\mathrm{e}}},
\end{gathered}
$$




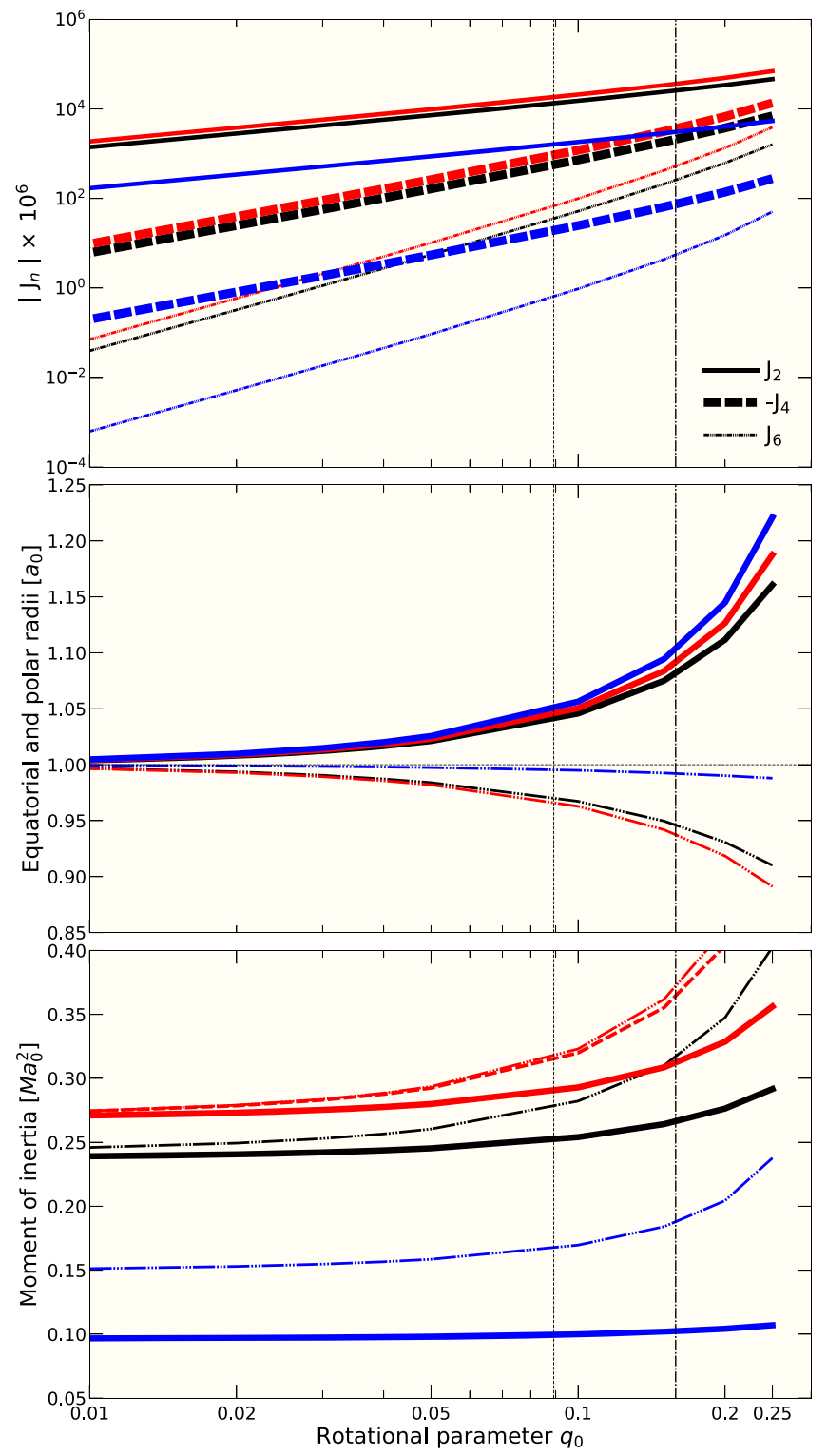

Figure 3. Models of three rotating planets without tidal perturbations: cold (black; $S=7.2 k_{b} / \mathrm{el}, a_{0}=0.876 R_{\mathrm{J}}$ ) and hot (red; $S=11.0 k_{b} / \mathrm{el}, a_{0}=2.604 R_{\mathrm{J}}$ ) Saturnmass planets and a cold Jupiter-mass planet (blue; $S=7.2 k_{b} / \mathrm{el}, a_{0}=1.004 R_{\mathrm{J}}$ ) are compared as function of $q_{0}$. The vertical dashed and dashed-dotted lines mark the $q_{\mathrm{e}}$ values of Jupiter and Saturn, respectively. The top panel shows the gravity harmonics $J_{2}, J_{4}$, and $J_{6}$ that scale like $q_{0}, q_{0}^{2}$, and $q_{0}^{3}$ for small $q_{0}$ values. The middle panel shows how the equatorial (solid) and polar (dashed-dotted) radii of these planets change with increasing rotation rate. The radii of the nonrotating planets, $a_{0}$, are used as normalization. In the bottom panel, we compare the CMS predictions (solid lines) for the normalized moment of inertia with the DarwinRadau expressions, one involving $J_{2}$ (Equation (10); dashed-dotted line) and the other relying on the oblateness (Equation (9); dotted line).

$$
x_{2}=\frac{5 q_{s}}{3 J_{2}+q_{s}}-1 \text { with } q_{s}=\frac{\omega^{2} s^{3}}{G M}=q_{\mathrm{e}} \frac{s^{3}}{a_{\mathrm{e}}^{3}},
$$

where $a_{\mathrm{e}}, c$, and $s$ are the equatorial, polar, and sphericalized radii $\left(\frac{4}{3} \pi s^{3}\right.$ equals the planet volume). The quantity $x$ can either be derived from the oblateness, $f$, or expressed in terms of the parameters $J_{2}$ and $q_{s}$. Both expressions give similar results unless the density contrast between the core and envelope is too large, as we see for the hot Saturn-mass planet in Figure 3. In this case, both Darwin-Radau expressions overestimate the moment of inertia by $\sim 50 \%$, even in the limit of a slowly rotating planet. In this limit, the Darwin-Radau results agree fairly well with the CMS predictions of the colder Saturn- and Jupiter-mass planets. The equatorial radii of these two planets are 0.88 and $1.0 R_{\mathrm{J}}$, while the hot Saturn-mass planet is significantly inflated $\left(a=2.6 R_{\mathrm{J}}\right)$, which explains the breakdown of the Darwin-Radau expression. Furthermore, one should also be cautious in applying the Darwin-Radau approximation to fast-rotating planets like Saturn and Jupiter, with $q_{\mathrm{e}}=0.158$ and 0.0892 , respectively. In this case, $q_{\mathrm{e}}$ is no longer a small parameter, and the Darwin-Radau assumptions break down.

In Figure 4, we compare the Love numbers, $k_{n m}$, of six hypothetical planets, considering three different masses 0.3 , 1.0 , and $10.0 M_{\mathrm{J}}$ with a cold or hot $\left(S=7.2\right.$ and $\left.11.0 k_{b} / \mathrm{el}\right)$ interior. All have $10 M_{\oplus}$ cores. In the limit of slow rotation, for a given $n$, we find that nonzero $k_{n m}$ values of any $m$ all approach a common value, as expected since angular dependence disappears for a nonrotating planet. All Love numbers rise with increasing rotation rate. However, this rise is very small for the hot Saturn-mass planets, which exhibit the smallest Love numbers, followed by the hot Jupiter-mass planets. On the other hand, the cold Jupiter-mass planets exhibit the largest Love numbers of all six planets, except for very large $q_{0}$ values where the cold $10 M_{\mathrm{J}}$ planet shows a larger response. For the hot planets $\left(S=11 k_{b} / \mathrm{el}\right)$, the Love number increases with rising planet mass, while no simple trend appears for three colder planets.

In Figure 5, we compare the Love number, $k_{22}$, as a function of various parameters in order to motivate $k_{22} \approx 0.6$ as a plausible maximum for slowly rotating planets. In the top panel, we study the dependence on planet mass. For cold planets $\left(S=7.2 k_{b} / \mathrm{el}\right), k_{22}$ assumes a maximum value of 0.603 for a $1 M_{\mathrm{J}}$ planet. This planet has a radius of $1.026 R_{\mathrm{J}}$, which is close to the maximal radius of $1.069 R_{\mathrm{J}}$ that emerges for a $3 M_{\mathrm{J}}$ planet.

In the middle panel, we plot $k_{22}$ as a function of the envelope entropy, $S$. With increasing $S$, the envelope becomes less dense and thus shows a reduced tidal response. When the entropy of a $1 M_{\mathrm{J}}$ planet is reduced from $S=7.2$ to $6.84 k_{b} / \mathrm{el}$, the typical value for the Saturn $k_{22}$ increases from 0.603 to 0.618 . Since this represents a cold planet, we argue that 0.6 is still a reasonable upper bound for the $k_{22}$ of slowly rotating hot Jupiters.

In the bottom panel, we study the dependence of $k_{22}$ as a function of core mass fraction while keeping the total planet mass fixed at 1.0 and $10.0 M_{\mathrm{J}}$. As expected, $k_{22}$ decreases with increasing core mass fraction because it concentrates more mass in the planet's center, where it responds less to tidal perturbations. It is this dependence of $k_{22}$ on core mass that will enable the inference of an exoplanet's core mass with future transit measurements (e.g., Batygin et al. 2009; Ragozzine \& Wolf 2009).

\subsection{Results for Selected Exoplanets}

Meaningful models of the interior structure of a given planet require constraints on both planetary mass and radius, which are determined by independent observation techniques. Table 2 summarizes the input parameters for eight exoplanets considered here. Three of the selected planets, HAT-P-13b (Buhler et al. 2016; Hardy et al. 2017), WASP-18b (Csizmadia et al. 2019), and 

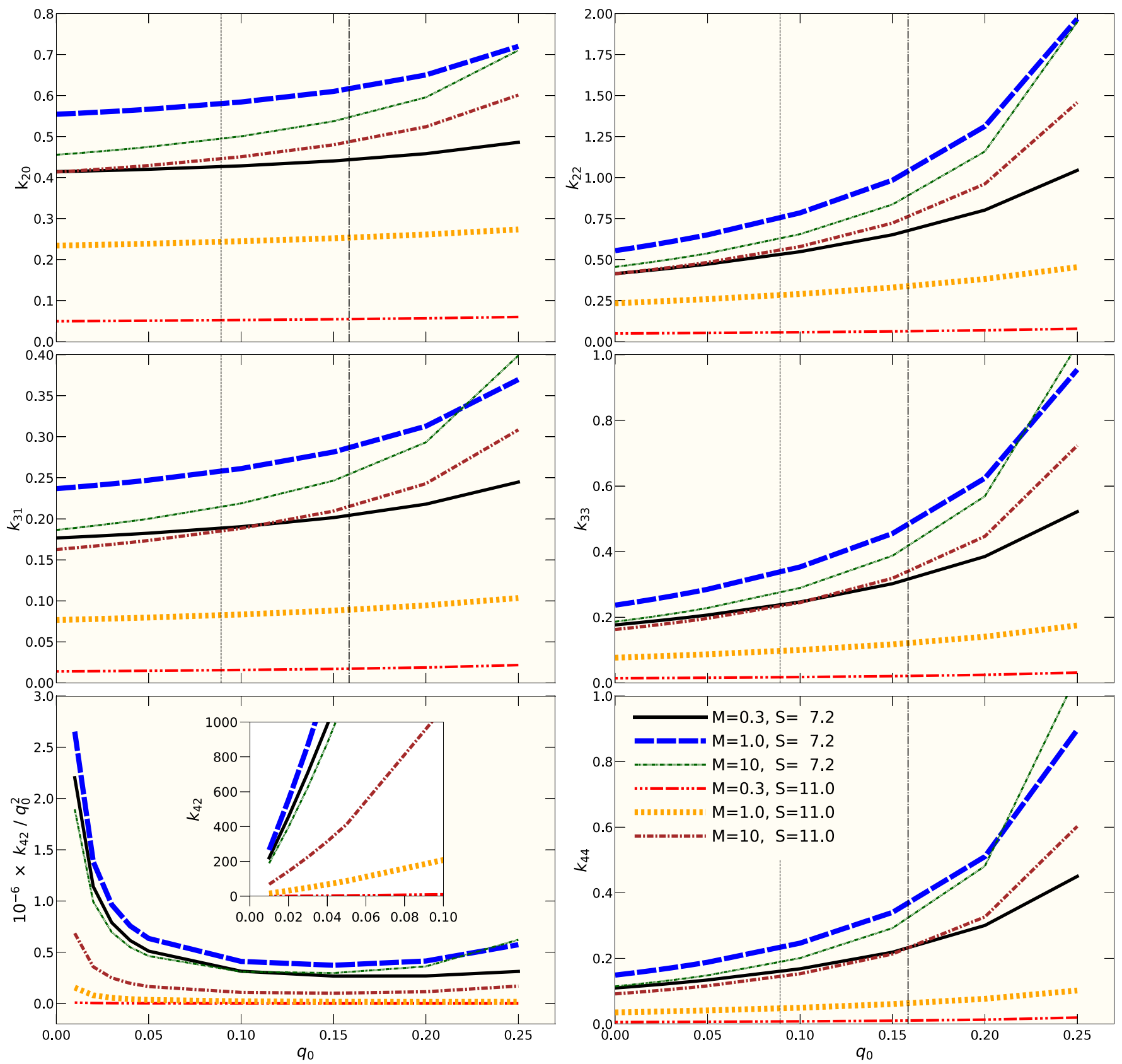

Figure 4. Tidal Love numbers, $k_{n m}$, for six planet models are shown as function of $q_{0}$. The masses and interior entropies are given in the legend in the bottom right panel. In the bottom left panel, $k_{42}$ has been divided by $q_{0}^{2}$, while the inset shows the original $k_{42}$. The vertical lines mark the $q_{\mathrm{e}}$ values of Jupiter and Saturn as in Figure 3.

WASP-4b (Bouma et al. 2019), have reported observational constraints on $k_{22}$, while WASP-12b (Campo et al. 2011), WASP$103 \mathrm{~b}$ (Akinsanmi et al. 2019), and WASP-121b (Hellard et al. 2020) have each been invoked in studies of the detectability of $k_{22}$. Figure 6 shows reported $k_{22}$ observations compared to the limits on $k_{22}$ we find for all eight selected exoplanets.

Modeling a realistic interior structure of a giant planet necessarily involves calculating its thermal structure. For the two-layer models considered, it is natural to parameterize this in terms of the specific entropy of the envelope, $S$. Cool giant planets like Jupiter and Saturn begin their life with a high specific entropy (from their formation heat) and gradually cool over time (e.g., Fortney et al. 2007). Giant planets whose incident flux exceeds $2 \times 10^{8} \mathrm{erg} \mathrm{s}^{-1} \mathrm{~cm}^{-2}$, however, exhibit large radii indicative of hotter interiors than are expected from the physical processes seen in their cooler cousins (Demory \& Seager 2011; Miller \& Fortney 2011).

This hot Jupiter inflation effect may be modeled as an additional heat source within the planet that varies with the incident stellar flux (Thorngren \& Fortney 2018; Sarkis et al. 2021). As they evolve, these planets will, therefore, approach a steady state where the energy flux out of the interior (parameterized by the intrinsic temperature $T_{\text {int }}$ ) is equal to the anomalous heating (Thorngren et al. 2019). Using the atmosphere models of Fortney et al. (2009), we can relate the envelope's specific entropy to $T_{\mathrm{int}}$. Then we apply the results of 

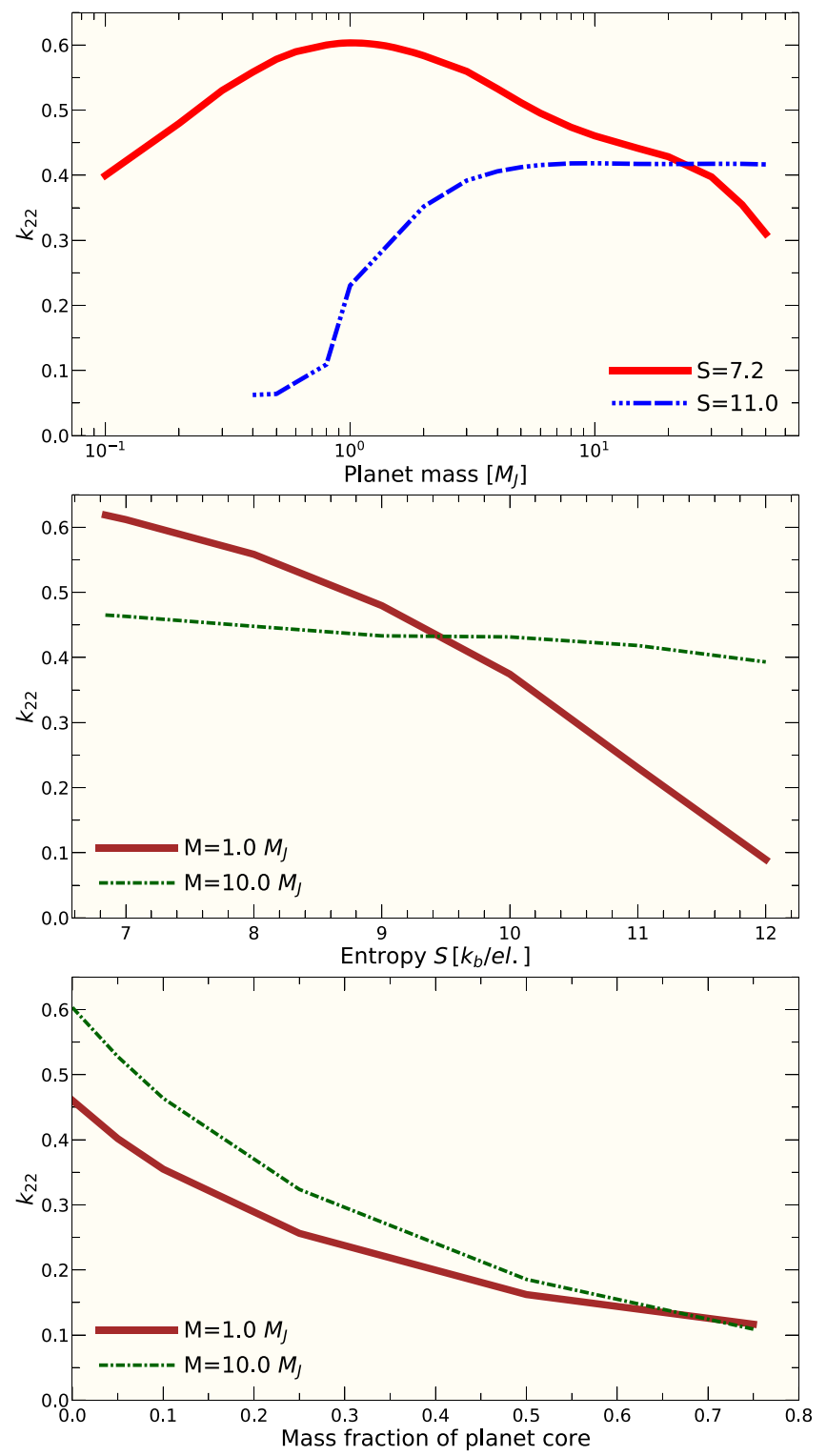

Figure 5. For slowly rotating planets, the three panels show the tidal coefficient $k_{22}$ as a function of planet mass (top); the envelope entropy, $S$ (middle); and core mass (bottom).

Thorngren \& Fortney (2018), which relate the anomalous heating to the incident flux. The final product is a relationship between the specific entropy of the planet with the incident flux onto the planet (Figure 7). This is helpful because the entropy is extremely difficult to measure observationally, but the incident flux is easily calculated from stellar and orbital properties. To aide the reader, we represent fluxes as the corresponding equilibrium temperatures assuming zero albedo and full heat redistribution, $T_{\mathrm{eq}}^{4}=F /\left(4 \sigma_{b}\right)$, where $\sigma_{b}$ is the Stefan-Boltzmann constant.

Figure 7 shows the relationship between equilibrium temperature and entropy for planets with masses between 0.1 and $10 M_{\mathrm{J}}$ in thermal equilibrium with $T_{\text {eq }}$ between 1000 and $2500 \mathrm{~K}$. For planets within this range, the entropy is interpolated with $T_{\text {eq }}$ at a constant mass from the two $T_{\text {eq }}-S$ curves with the closest temperatures. More massive planets were considered by extrapolating the $T_{\text {eq }}-S$ curves as a function of $\log _{10}(M)$. Cooler planets were considered using a curve constant entropy of $S=7.2$

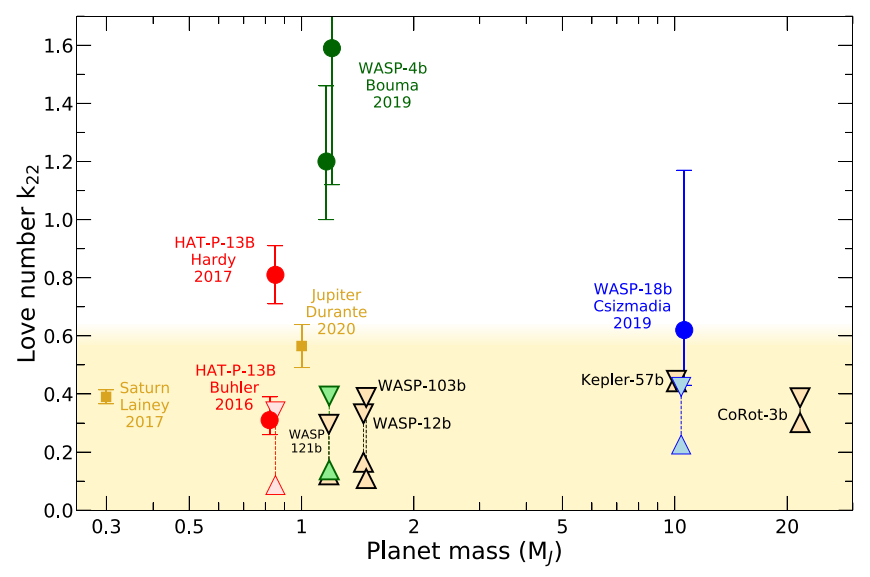

Figure 6. The triangles show the calculated limits on $k_{22}$ for the selected exoplanets in Table 2. The upper limit is a model without a central core, while the lower limit is a case with no heavy elements in the envelope $(Z=0)$. The filled circles represent exoplanets with reported $k_{22}$ observations. The shaded region represents values of $k_{22} \leqslant 0.6$ that can be explained in terms of a static tidal response.

$k_{\mathrm{B}} / \mathrm{el}$, which is the condition for the onset of helium rain as shown in Figure 1. The temperature for this "cold" curve is then defined by the reported effective temperatures for Jupiter and Saturn at the onset of helium rain by Mankovich \& Fortney (2020). However, planets with lower incoming stellar energy flux are likely to be further from the assumed equilibrium state. Our relationship between specific entropy and $T_{\text {eq }}$ relies on a fit to the observed population of hot Jupiters (in Thorngren \& Fortney 2018). As such, we have avoided extrapolating very far into regions of mass-flux space where few to no hot Jupiters are found. In general, this cutoff moves to higher masses as the temperature increases as a result of planet formation processes outside the scope of this paper.

Statistical uncertainties in the heating efficiency from Thorngren \& Fortney (2018) are approximately $0.5 \%$, which leads to an uncertainty in the resulting $T_{\text {int }}$ of around $35 \mathrm{~K}$, varying with the incident flux. However, these statistical uncertainties are less significant than the modeling uncertainty, which is much more difficult to quantify. An alternate approach was presented by Sarkis et al. (2021), who found broadly similar values for the heating efficiency, though their peak heating of $2.5 \%$ was at a higher temperature of $\sim 1860 \mathrm{~K}$. Thorngren \& Fortney (2018) found a peak of $2.5 \%$ at $1500-1600 \mathrm{~K}$. This difference appears to be the result of differences in the atmosphere models. Mollière et al. (2015) included the effects of $\mathrm{TiO}$ and VO species on upper atmosphere opacities, whereas Fortney et al. (2007) and therefore Thorngren \& Fortney (2018) did not. Both papers are fitting to the observed radius via the entropy, so the difference in the predicted entropy for a given planet likely does not differ as much as the heating efficiency. However, this fit relies on assumptions for a planet's composition (abundance of helium and heavier elements) and the EOS that defines isentropic paths in $P-T$ space and the corresponding density. Uncertainties in the EOSs of hydrogenhelium mixtures and their impact on giant-planet structure have been discussed by Saumon \& Guillot (2004), Militzer et al. (2016), and Helled et al. (2020).

The interior density profile is determined by the isentropic pressure-density curve derived from the EOS, which depends both on the entropy and on the heavy-element fraction of the envelope, $Z$. Given the observational constraints on mass and 
Table 2

Parameters for Selected Exoplanets

\begin{tabular}{|c|c|c|c|c|c|c|c|c|c|c|}
\hline Name & $\begin{array}{c}t_{\text {orbit }} \\
\text { (days) }\end{array}$ & $\begin{array}{c}R_{\text {orbit }} \\
\text { (au) }\end{array}$ & $e$ & $\begin{array}{c}M_{\mathrm{p}} \\
\left(M_{\mathrm{J}}\right)\end{array}$ & $\begin{array}{c}R_{\mathrm{p}} \\
\left(R_{\mathrm{J}}\right)\end{array}$ & $\begin{array}{c}M_{\mathrm{st}} \\
\left(M_{\odot}\right)\end{array}$ & $\begin{array}{c}R_{\mathrm{st}} \\
\left(R_{\odot}\right)\end{array}$ & $\begin{array}{c}T_{\text {eff,st }} \\
(\mathrm{K})\end{array}$ & $\begin{array}{c}T_{\text {eq,p }} \\
(\mathrm{K})\end{array}$ & $\begin{array}{c}S \\
\left(k_{B} / e^{-}\right)\end{array}$ \\
\hline HAT-P-13b ${ }^{\mathrm{a}}$ & 2.916 & 0.04269 & 0.0133 & 0.851 & 1.272 & 1.22 & 1.56 & 5653 & 1649 & 11.14 \\
\hline WASP- $18 b^{b}$ & 0.9415 & 0.02009 & 0.0091 & 10.40 & 1.191 & 1.22 & 1.23 & 6400 & 2416 & 10.92 \\
\hline WASP- $4 b^{c}$ & 1.338 & 0.02261 & 0 & 1.186 & 1.321 & 0.86 & 0.89 & 5400 & 1634 & 10.99 \\
\hline WASP-12b ${ }^{\mathrm{d}}$ & 1.091 & 0.02338 & 0 & 1.465 & 1.937 & 1.43 & 1.66 & 6360 & 2585 & 11.87 \\
\hline WASP- $121 b^{\mathrm{e}}$ & 1.275 & 0.02544 & 0 & 1.183 & 1.865 & 1.35 & 1.46 & 6459 & 2361 & 11.84 \\
\hline WASP-103b ${ }^{\mathrm{f}}$ & 0.9255 & 0.01987 & 0 & 1.49 & 1.528 & 1.22 & 1.44 & 6110 & 2509 & 11.81 \\
\hline Kepler-75b ${ }^{g}$ & 8.885 & 0.08164 & 0.57 & 10.1 & 1.05 & 0.91 & 0.89 & 5200 & 870 & 8.26 \\
\hline CoRoT- $3 b^{h}$ & 4.257 & 0.05738 & 0 & 21.66 & 1.01 & 1.37 & 1.56 & 6740 & 1695 & 9.90 \\
\hline
\end{tabular}

Notes. Here $T_{\text {eq,p }}$ assumes zero albedo, and $S$ is interpolated from the relationships in Figure 7 .

${ }^{\mathrm{a}}$ Winn et al. (2010).

b Shporer et al. (2019).

c Bouma et al. (2019).

d Chakrabarty \& Sengupta (2019).

e Delrez et al. (2016).

${ }^{f}$ Gillon et al. (2014).

g Bonomo et al. (2015).

h Deleuil et al. (2008).

radius, a two-layer model exoplanet can accommodate heavyelement mass in both the core and envelope. Figure 8 shows the density profiles of two end-member cases for each selected exoplanet. The first end-member is the case of a coreless model, which corresponds to a maximum value for $Z$ in the envelope. The second case is a model in which $Z=0$ in the envelope, corresponding to a maximum core mass. These are compared to two-layer analog models of Jupiter and Saturn, which match the observed $J_{2}$ for each (Iess et al. 2019; Durante et al. 2020), in addition to the mass and radius. Two-layer models are known to do a poor job of reproducing the full gravitational field of Jupiter and Saturn, since they ignore the redistribution of helium, as well as a possible "dilute" core (Wahl et al. 2017b; Mankovich \& Fuller 2021) or inhomogeneity of $Z$ across the helium rain layer (Miguel et al. 2016; Debras \& Chabrier 2019). For this reason, the two-layer analogs of Jupiter and Saturn have more massive central cores and require either negative values of $Z$ or significantly higher temperatures than more complicated interior models. In Figure 8, there are evident influences from both planet mass and equilibrium temperature, with the very massive CoRoT-3b exhibiting the highest densities in the deep envelope, and the highly irradiated WASP-12b and WASP-121b exhibiting far more extended envelopes with notably lower density gradients in the outer portion of the planet.

As a consequence of their assumed tidally locked state, both the tidal and rotational parameters, $q_{0}$ and $q_{\mathrm{tid}}$, are tied to the orbital distance of the planet. Table 3 presents $q_{0}$ and $q_{\text {tid }}$ for the eight selected exoplanets in the assumed 1:1 resonance locked state, along with results from the computed tidal responses, $k_{22}$ and $k_{20}$, and the shape of the two end-member interior structures. For the planet shape, we report the three principle axis lengths: $a$, equatorial radius along the star-planet axis; $b$, equatorial radius perpendicular to the star-planet axis; and $c$, polar radius along the rotation axis. Additionally, we report the prolateness and oblateness, defined as

$$
f_{a c}=\frac{a-c}{a} \text { and } f_{b c}=\frac{b-c}{b},
$$

respectively. Although the aforementioned shape parameters are commonly used to describe triaxial ellipsoids, we note that the spheroidal surface predicted by CMS represents a more general shape. In fact, the calculated surface is only an exact ellipsoid in the case of a constant density planet (Wahl et al. 2017a).

HAT-P-13b and the five planets selected from the WASP catalog exhibit extremely short orbital periods and are expected to be tidally locked. Kepler-75b and CoRoT-3b orbit much more distantly, leading to much smaller values of $q_{0}$. For reasonable values of tidal quality factor, $Q$, the two more distantly orbiting planets are also likely to be tidally locked. Alternatively, if, like Jupiter and Saturn, these planets' rotation rates have not been significantly slowed by tidal torques, then the values of $q_{0}$ could be orders of magnitude higher than for the tidally locked state reported here.

For all eight exoplanets, the end-member interior structure case with $Z=0$ in the envelope determines the maximum core mass and radii and corresponds to a minimum prediction of $f_{a c}$ and $f_{b c}$, as well as minimal values of $k_{20}$ and $k_{22}$. Conversely, the case with no central core yields the maximum $Z$ in the envelope, as well as maximum values for $f_{a c}, f_{b c}, k_{22}$, and $k_{20}$. While not explicitly considered here, planets with dilute cores (Wahl et al. 2017b) are expected to exhibit tidal responses between these two end-member cases, since they represent an intermediate degree of concentration of heavy elements between a fully mixed planet and one with all heavy elements in a dense, central core.

While the central concentration of heavy elements has the strongest influence on $k_{22}$ for a given planet, the planet mass and equilibrium temperature are significant over the full parameter space of giant exoplanets. Figure 9 shows five mass-radius relationships calculated for two-layer models with a constant $10 M_{\oplus}$ core and $Z=0$ in the envelope, along with the corresponding $k_{22}$ for a tidal response in the linear regime, $q_{0}<1 \times 10^{-5}$ and $q_{\text {tid }}>-1 \times 10^{-5}$. The blue curve shows the mass-radius relationship for "cold" exoplanets with $S=7.2$ $k_{\mathrm{B}} / \mathrm{el}$. This shows the characteristic trend with radius first increasing with mass to a maximum of $\sim 1.05 R_{\mathrm{J}}$ at $\sim 3 M_{\mathrm{J}}$ and then decreasing as additional mass leads to a shrinking radius due to compaction of the hydrogen-helium envelope. The calculated $k_{22}$ follows a qualitatively similar trend, with a 


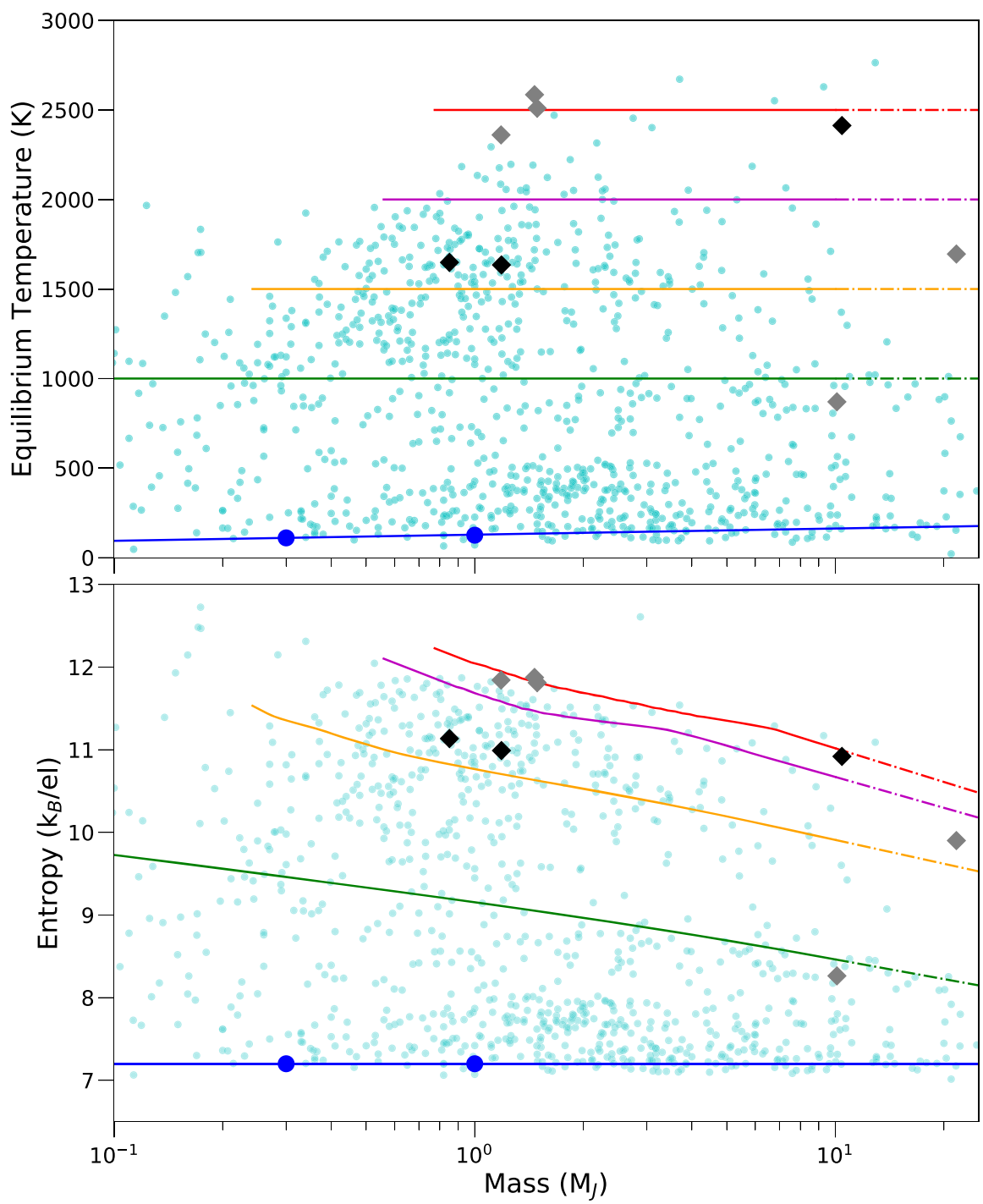

Figure 7. Equilibrium temperature (top) and entropy (bottom) relationship calculated for thermal equilibrium as a function of planet mass, shown as solid lines at $T_{\mathrm{eq}}=1000,1500,2000$, and $2500 \mathrm{~K}$. Extrapolated $T_{\mathrm{eq}}-S$ trends for planet masses $>10 M_{\mathrm{J}}$ are shown with dashed-dotted lines. The condition for the onset of helium rain in Saturn and Jupiter is denoted by blue circles. Diamonds are selected exoplanets in Table 2 with (black) and without (gray) published $k_{2}$ observations. Planets in NASA Exoplanet Science Institute (2011) with the necessary parameters are shown in cyan under the same assumptions of thermal equilibrium.

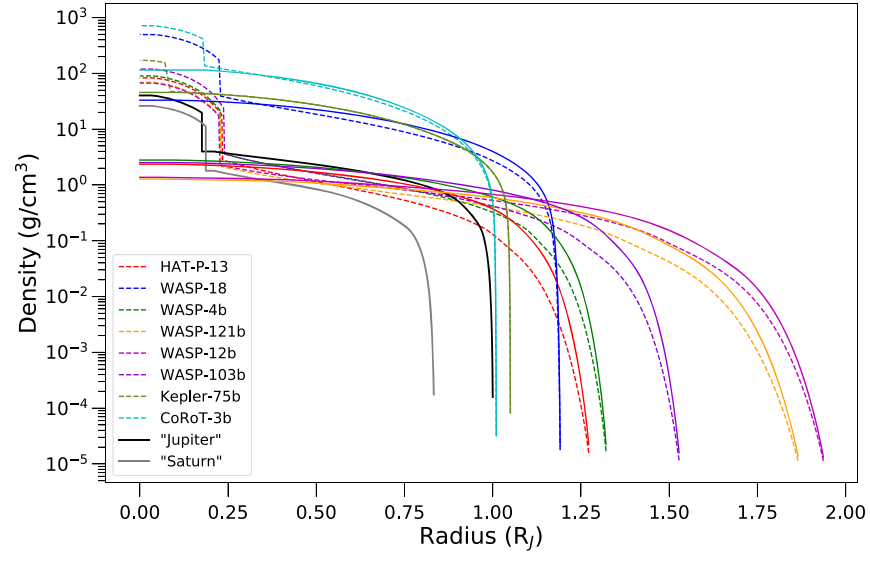

Figure 8. Density as a function of radius from the center of the planet for eight exoplanets and analogs of Jupiter and Saturn. For each exoplanet, two endmember interior structures are shown: fully mixed with no core and maximum envelope $Z$ (solid) and fully separated with maximum core mass and envelope $Z=0$ (dashed). The Saturn and Jupiter analogs have $M_{c}$ and $Z$ chosen to match the observed $J_{2}$ (Iess et al. 2019; Durante et al. 2020).
$0.1 M_{\mathrm{J}}$ planet exhibiting a $k_{22}$ of $\sim 0.2$, increasing with mass to a maximum at $\sim 0.56$ at $\sim 1.5 M_{\mathrm{J}}$, and then decreasing to $\sim 0.46$ at $10 M_{\mathrm{J}}$.

The mass-radius relationship for a constant $T_{\text {eq }}=1000 \mathrm{~K}$ has a quite different appearance, with an inflated $0.1 M_{\mathrm{J}}$ planet first decreasing with mass, flattening out at $\sim 0.6 M_{\mathrm{J}}$, and then decreasing further for $M>2 M_{\mathrm{J}}$. The slope at low masses becomes steeper with increasing $T_{\text {eq }}$, and the radius decreases monotonically, with an inflection point moving to higher masses for higher $T_{\text {eq }}$. Meanwhile, the calculated $k_{22}$ decreases with increasing $T_{\text {eq }}$, with the maximum $k_{22}$ for a given massradius relationship shifting to higher masses. While an increasing envelope $Z$ leads to a decrease in radius, it has a somewhat less intuitive influence on $k_{22}$. For a colder Jupitermass planet with $S=7.20 k_{\mathrm{B}} / \mathrm{el}$, increasing $Z$ leads to a very slight decrease in $k_{22}$, while an identical-mass planet with $T_{\text {eq }}=2500 \mathrm{~K}$ exhibits a more substantial increase in $k_{22}$ with $Z$.

The observed $k_{22}$ values for exoplanets, summarized in Figure 6, cover a wider range of values, most of which are larger than our models can account for with a two-layer interior 
Table 3

Predicted Shape and Tidal Response for Selected Exoplanets Assuming a Tidally Locked State and a Two-layer Interior Model with $N=1025$ CMS Layers

\begin{tabular}{|c|c|c|c|c|c|c|c|c|c|c|c|c|c|c|}
\hline $\begin{array}{l}\text { Planet } \\
\text { Name }\end{array}$ & $q_{\mathrm{rot}}$ & $q_{\text {tid }}$ & $Z$ & $\begin{array}{c}M_{c} \\
{\left[M_{\mathrm{J}}\right]}\end{array}$ & $\begin{array}{c}R_{c} \\
{\left[R_{\mathrm{J}}\right]}\end{array}$ & $k_{22}$ & $k_{20}$ & $\begin{array}{c}a \\
{\left[R_{\mathrm{J}}\right]}\end{array}$ & $\begin{array}{c}b \\
{\left[R_{\mathrm{J}}\right]}\end{array}$ & $\begin{array}{c}c \\
{\left[R_{\mathrm{J}}\right]}\end{array}$ & $f_{a c}$ & $f_{b c}$ & $\Delta R$ & $C /\left(M a_{0}^{2}\right)$ \\
\hline HAT-P-13b & $4.06 \mathrm{e}-2$ & -0.0122 & $\begin{array}{c}0.389 \\
0\end{array}$ & $\begin{array}{c}0 \\
0.387\end{array}$ & $\begin{array}{c}0 \\
0.232\end{array}$ & $\begin{array}{c}0.34 \\
0.0866\end{array}$ & $\begin{array}{c}0.337 \\
0.0862\end{array}$ & $\begin{array}{l}1.27600 \\
1.27956\end{array}$ & $\begin{array}{l}1.26561 \\
1.27103\end{array}$ & $\begin{array}{l}1.26223 \\
1.26825\end{array}$ & $\begin{array}{l}1.08 \mathrm{e}-2 \\
8.84 \mathrm{e}-3\end{array}$ & $\begin{array}{l}2.67 e-3 \\
2.18 \mathrm{e}-3\end{array}$ & $\begin{array}{l}6.39 \mathrm{e}-3 \\
1.86 \mathrm{e}-3\end{array}$ & $\begin{array}{l}0.220 \\
0.108\end{array}$ \\
\hline WASP-18b & $2.61 \mathrm{e}-2$ & $-7.78 \mathrm{e}-2$ & $\begin{array}{c}0.212 \\
0\end{array}$ & $\begin{array}{c}0 \\
2.53\end{array}$ & $\begin{array}{c}0 \\
0.225\end{array}$ & $\begin{array}{l}0.424 \\
0.226\end{array}$ & $\begin{array}{l}0.422 \\
0.225\end{array}$ & $\begin{array}{l}1.19632 \\
1.19595\end{array}$ & $\begin{array}{l}1.18967 \\
1.19022\end{array}$ & $\begin{array}{l}1.18748 \\
1.18832\end{array}$ & $\begin{array}{l}7.39 \mathrm{e}-3 \\
6.38 \mathrm{e}-3\end{array}$ & $\begin{array}{l}1.84 \mathrm{e}-3 \\
1.59 \mathrm{e}-3\end{array}$ & $\begin{array}{l}2.04 \mathrm{e}-3 \\
1.45 \mathrm{e}-3\end{array}$ & $\begin{array}{l}0.240 \\
0.177\end{array}$ \\
\hline WASP-4b & 0.0155 & -0.0464 & $\begin{array}{c}0.308 \\
0\end{array}$ & $\begin{array}{c}0 \\
0.409\end{array}$ & $\begin{array}{c}0 \\
0.227\end{array}$ & $\begin{array}{l}0.393 \\
0.139\end{array}$ & $\begin{array}{l}0.381 \\
0.136\end{array}$ & $\begin{array}{l}1.35600 \\
1.35369\end{array}$ & $\begin{array}{l}1.31136 \\
1.31667\end{array}$ & $\begin{array}{l}1.29796 \\
1.30546\end{array}$ & $\begin{array}{l}4.28 \mathrm{e}-2 \\
3.56 \mathrm{e}-2\end{array}$ & $\begin{array}{l}1.02 \mathrm{e}-2 \\
8.51 \mathrm{e}-3\end{array}$ & $\begin{array}{l}1.25 \mathrm{e}-2 \\
7.59 \mathrm{e}-3\end{array}$ & $\begin{array}{l}0.230 \\
0.137\end{array}$ \\
\hline WASP-121b & 0.0481 & -0.144 & $\begin{array}{c}0.238 \\
0\end{array}$ & $\begin{array}{c}0 \\
0.313\end{array}$ & $\begin{array}{c}0 \\
0.228\end{array}$ & $\begin{array}{l}0.296 \\
0.122\end{array}$ & $\begin{array}{l}0.275 \\
0.115\end{array}$ & $\begin{array}{l}2.02633 \\
2.03212\end{array}$ & $\begin{array}{l}1.82110 \\
1.84487\end{array}$ & $\begin{array}{l}1.77207 \\
1.79998\end{array}$ & $\begin{array}{l}0.125 \\
0.114\end{array}$ & $\begin{array}{l}2.69 \mathrm{e}-2 \\
2.43 \mathrm{e}-2\end{array}$ & $\begin{array}{l}3.82 \mathrm{e}-2 \\
2.34 \mathrm{e}-2\end{array}$ & $\begin{array}{l}0.200 \\
0.130\end{array}$ \\
\hline WASP-12b & 0.0594 & -0.178 & $\begin{array}{c}0.19 \\
0\end{array}$ & $\begin{array}{c}0 \\
0.294\end{array}$ & $\begin{array}{c}0 \\
0.223\end{array}$ & $\begin{array}{l}0.332 \\
0.165\end{array}$ & $\begin{array}{l}0.304 \\
0.154\end{array}$ & $\begin{array}{l}2.16934 \\
2.17185\end{array}$ & $\begin{array}{l}1.88228 \\
1.90686\end{array}$ & $\begin{array}{l}1.82032 \\
1.84932\end{array}$ & $\begin{array}{l}0.161 \\
0.149\end{array}$ & $\begin{array}{l}3.29 \mathrm{e}-2 \\
3.02 \mathrm{e}-2\end{array}$ & $\begin{array}{l}4.64 \mathrm{e}-2 \\
3.15 \mathrm{e}-2\end{array}$ & $\begin{array}{l}0.206 \\
0.150\end{array}$ \\
\hline WASP-103b & 0.0399 & -0.119 & $\begin{array}{c}0.347 \\
0\end{array}$ & $\begin{array}{c}0 \\
0.589\end{array}$ & $\begin{array}{c}0 \\
0.237\end{array}$ & $\begin{array}{l}0.388 \\
0.109\end{array}$ & $\begin{array}{l}0.362 \\
0.104\end{array}$ & $\begin{array}{l}1.64989 \\
1.63427\end{array}$ & $\begin{array}{l}1.50378 \\
1.51422\end{array}$ & $\begin{array}{l}1.46720 \\
1.48355\end{array}$ & $\begin{array}{c}0.111 \\
9.22 \mathrm{e}-2\end{array}$ & $\begin{array}{l}2.43 e-2 \\
2.03 e-2\end{array}$ & $\begin{array}{l}2.87 \mathrm{e}-2 \\
1.95 \mathrm{e}-2\end{array}$ & $\begin{array}{l}0.223 \\
0.117\end{array}$ \\
\hline Kepler-75b & $2.07 \mathrm{e}-5$ & $-6.15 e-5$ & $\begin{array}{c}4.21 \mathrm{e}-3 \\
0\end{array}$ & $\begin{array}{c}0 \\
4.40 \mathrm{e}-2\end{array}$ & $\begin{array}{c}0 \\
7.11 \mathrm{e}-2\end{array}$ & $\begin{array}{l}0.447 \\
0.442\end{array}$ & $\begin{array}{l}0.447 \\
0.442\end{array}$ & $\begin{array}{l}1.04978 \\
1.04987\end{array}$ & $\begin{array}{l}1.04973 \\
1.04982\end{array}$ & $\begin{array}{l}1.04971 \\
1.04981\end{array}$ & $\begin{array}{l}5.91 e-5 \\
6.00 e-5\end{array}$ & $\begin{array}{l}1.52 \mathrm{e}-5 \\
1.52 \mathrm{e}-5\end{array}$ & $\begin{array}{l}2.64 \mathrm{e}-4 \\
1.76 \mathrm{e}-4\end{array}$ & $\begin{array}{l}0.246 \\
0.244\end{array}$ \\
\hline CoRoT-3b & $3.74 \mathrm{e}-5$ & $-1.11 \mathrm{e}-4$ & $\begin{array}{c}8.32 \mathrm{e}-2 \\
0\end{array}$ & $\begin{array}{c}0 \\
2.30\end{array}$ & $\begin{array}{c}0 \\
0.178\end{array}$ & $\begin{array}{l}0.387 \\
0.301\end{array}$ & $\begin{array}{l}0.387 \\
0.301\end{array}$ & $\begin{array}{l}1.00894 \\
1.00992\end{array}$ & $\begin{array}{l}1.00886 \\
1.00984\end{array}$ & $\begin{array}{l}1.00883 \\
1.00982\end{array}$ & $\begin{array}{c}1.02 \mathrm{e}-4 \\
9.6 \mathrm{e}-5\end{array}$ & $\begin{array}{l}2.58 \mathrm{e}-5 \\
2.48 \mathrm{e}-5\end{array}$ & $\begin{array}{l}1.14 \mathrm{e}-3 \\
1.67 \mathrm{e}-4\end{array}$ & $\begin{array}{l}0.232 \\
0.207\end{array}$ \\
\hline
\end{tabular}

Note. For every planet, the top line is for the fully mixed case with no central core. The bottom line corresponds to the fully separated case with envelope $Z=0$ and maximum core mass. 

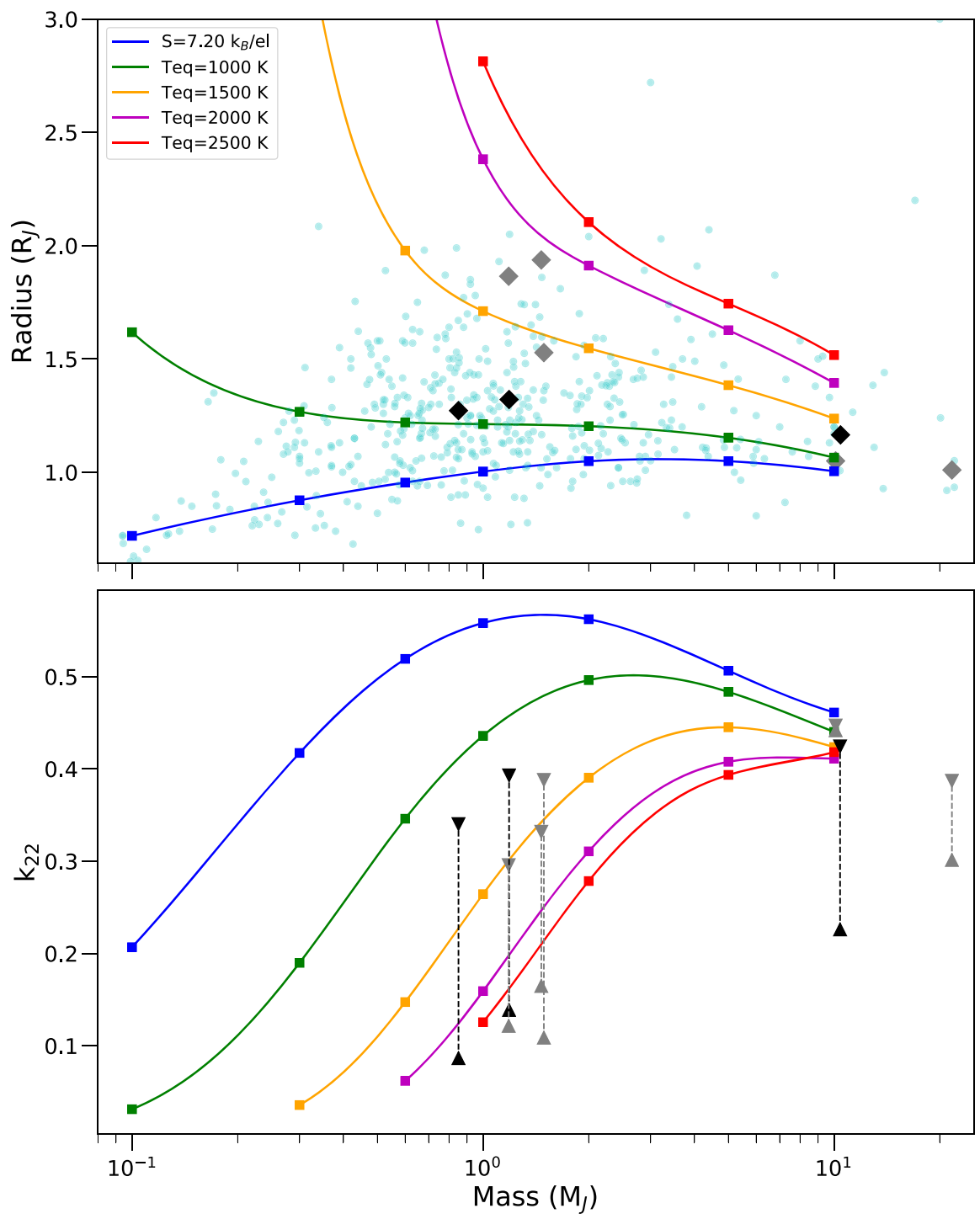

Figure 9. (Top) Mass-radius relationship for two-layer interior models with $M_{c}=10 M_{\oplus}, Z=0$, and constant $T_{\mathrm{eq}}$ between 1000 and $2500 \mathrm{~K}$ (red, magenta, yellow, and green squares) and for a constant entropy $S=7.2 \mathrm{k}_{\mathrm{B}} / \mathrm{el}$ (blue squares). Shown for comparison are masses and radii of confirmed exoplanets (cyan circles; NASA Exoplanet Science Institute 2011) and selected exoplanets (black and gray diamonds). (Bottom) Corresponding Love number, $k_{22}$, for mass-radius curves. The predicted range of $k_{22}$ for selected exoplanets is shown as in Figure 6.

structure and static tidal response. The Hardy et al. (2017) observation of HAT-P-13b and the Bouma et al. (2019) observation of WASP-4b are both significantly larger than our model predictions, even considering the reported uncertainties. They are, in fact, larger than the maximum of $\sim 0.6$ for any combination of parameters considered, with the possible exception of models undergoing extremely fast rotation. Our calculated range for WASP-18b has a maximum quite close to the lower limit of their prediction with the reported uncertainty (Csizmadia et al. 2019). Given the uncertainties on exoplanet mass, radius, and equilibrium temperature, the observation may, therefore, be compatible with the static tidal response of a coreless planet or one in which the core is a small fraction of the planet mass.

The observation of HAT-P-13b by Buhler et al. (2016) is the only observation showing significant overlap with the range of $k_{22}$ predicted here. Figure 10 demonstrates how $Z, k_{22}$, and $f$ vary as a function of core mass for our models of HAT-P13b and WASP$121 \mathrm{~b}$. The $k_{22}$ observed by Buhler et al. (2016) is consistent with a model planet ranging from no core to $\sim 0.075 M_{\mathrm{J}}\left(\sim 24 M_{\oplus}\right)$ and $Z$ between $\sim 0.34$ and 0.39 . It would, therefore, suggest that HAT-P$13 \mathrm{~b}$ has both a more massive core and an envelope more enriched in heavy elements than Jupiter (Miguel et al. 2016; Wahl et al. 2017b). The magnitudes of the prolateness and oblateness are governed primarily by $q_{0}$ and $q_{\text {tid }}$, but both show an $18 \%$ decrease between the fully mixed and fully separated HAT-P-13b models. The hotter, more expanded WASP-121b exhibits a qualitatively similar dependence on $M_{c}$ but with the observed mass and radius permitting a narrower range of $Z$ and $M_{\mathrm{c}}$ and, consequently, a smaller range of $k_{22}$.

In the linear regime, the static tidal response is fully determined by the density profile, and $k_{22}=k_{20}$ (Wahl et al. 2017a). Figure 11 demonstrates that the simulations for the various mass-radius relationships calculated with low $q_{0}$ and $q_{\text {tid }}$ precisely follow the relationship $k_{22}=3 J_{2} / q_{0}$. For larger values of $q_{0}$ and $q_{\text {tid }}$, nonlinearity in the tidal response leads to splitting between Love numbers $k_{22}$ and $k_{20}$. Jupiter and Saturn are in a regime with $q_{0} \gg q_{\text {tid }}$ and exhibit significant deviation from the linear regime 


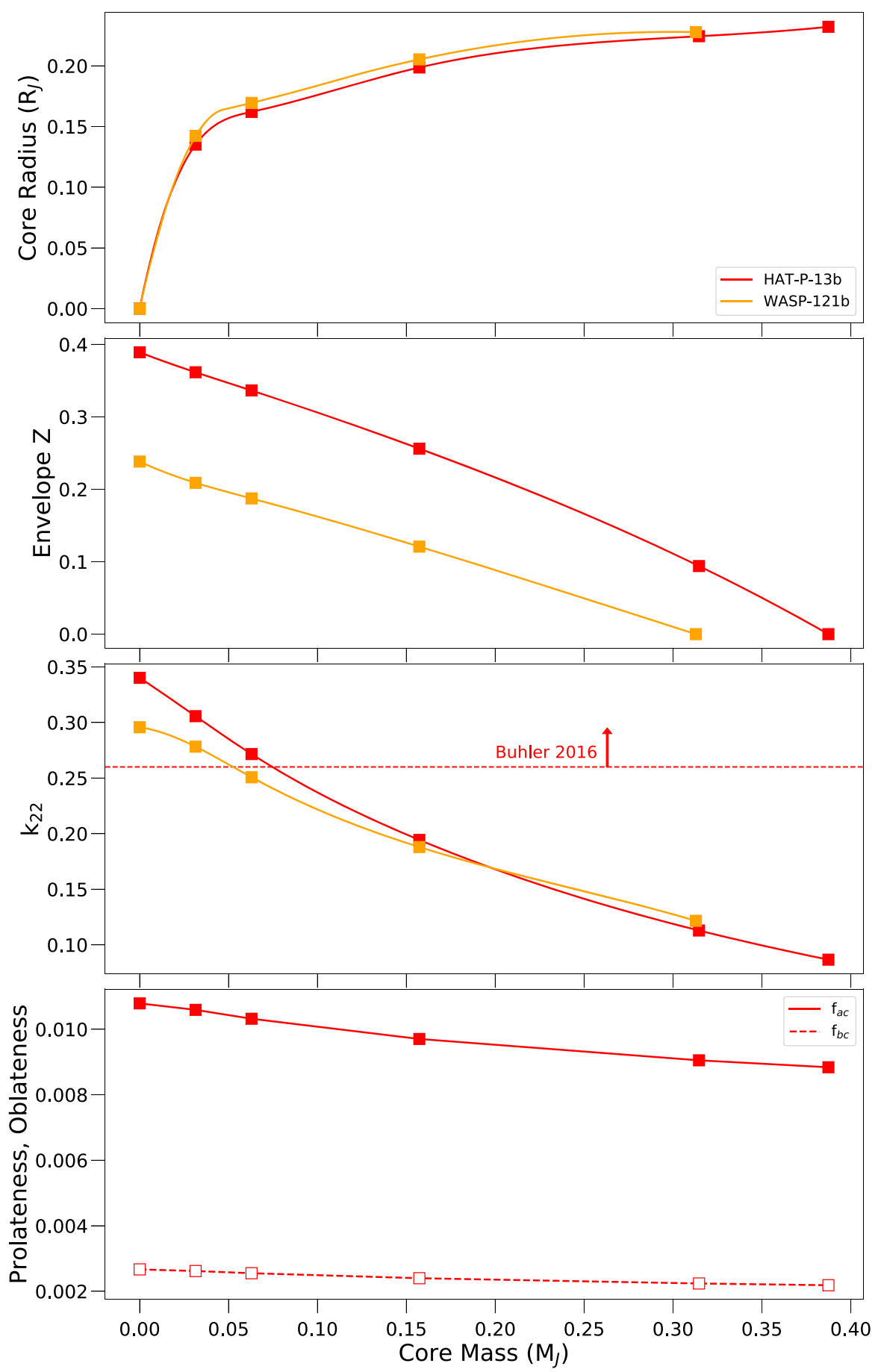

Figure 10. Variation of two-layer model features for HAT-P-13b and WASP-121b with core mass. First panel: core radius; second panel: heavy-element fraction, $Z$, of the envelope; third panel: Love number, $k 22$; fourth panel: prolateness, $f_{a c}$ (solid), and oblateness, $f_{b c}$ (dashed). The horizontal line in the third panel shows the minimum value consistent with the observed $k_{22}$ from Buhler et al. (2016).

relationship (Lainey et al. 2017, 2020; Wahl et al. 2017a, 2020; Durante et al. 2020). For hot Jupiters, $q_{0}$ is limited to being of a similar order of magnitude as $q_{\text {tid }}$ due to tidal locking, which means that significant deviations from the linear relationship occur only in the most extreme cases. Of the selected exoplanets, WASP-12b, WASP-103b, and WASP-121b all have $k_{22}$ values enhanced by more than $10 \%$ from $3 J_{2} / q_{0}$, with WASP-4b a slightly lesser $\sim 8 \%$ deviation. The largest deviation of $\sim 19 \%$ is found for a coreless WASP-12 model. Thus, we find that in the most extreme cases, tidally locked hot Jupiters can exhibit appreciably nonlinear tidal responses, though still to a lesser extent than the faster-rotating solar system giants. Conversely, nonlinearities can be safely ignored for exoplanets with $q_{0} \ll 0.01$.

Figure 12 shows the influence of $q_{0}$ on the second-order Love numbers, $k_{22}$ and $k_{20}$, while maintaining $q_{\text {tid }}$ at the value in Table 2 for the HAT-P-13b and WASP-121b models. As $q_{0}$ is increased, $k_{22}$ and $k_{20}$ split from their initial degenerate state. While this suggests that an observed $k_{22}$ as large as Hardy et al. (2017) might be possible for the static tidal response of a planet of HAT-P-13b's interior parameters, it would require $q_{0} \sim 0.2$, 


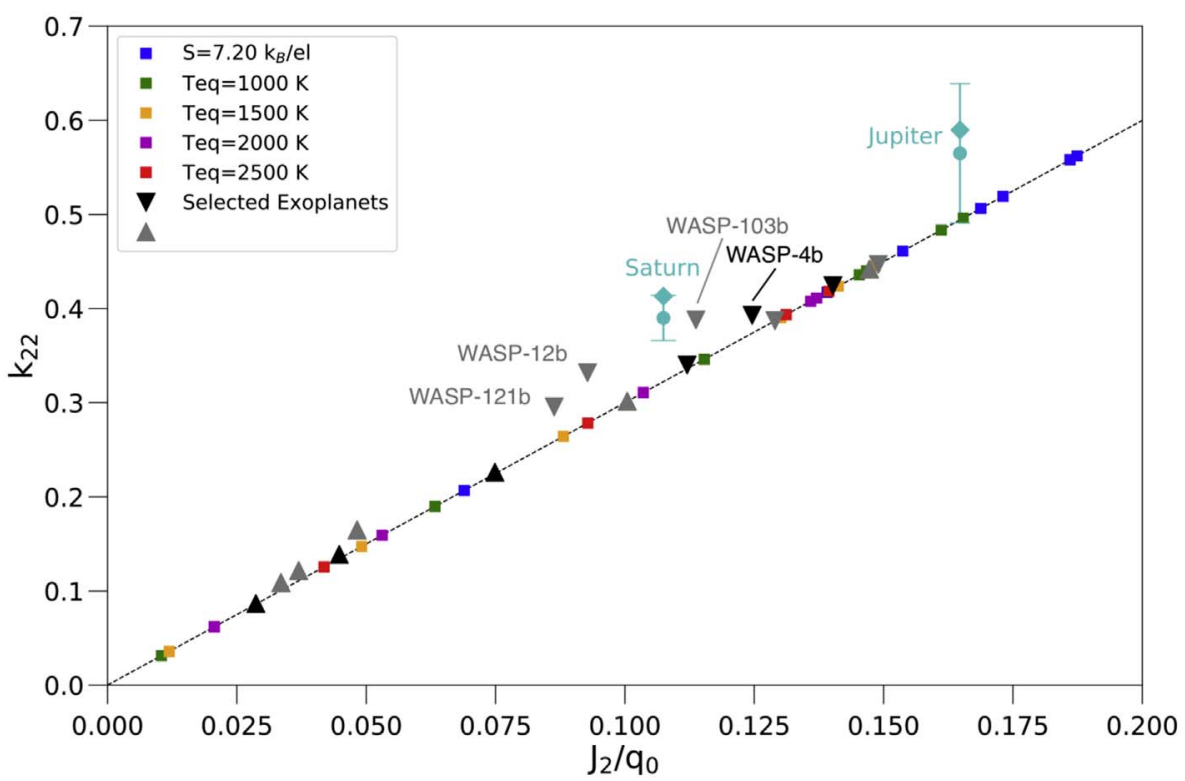

Figure 11. Comparison of the tidal response to the expected linear regime response. The dotted black line shows the relation $k_{22}=3 J_{2} / q_{0}$. Colored squares show results from the mass-radius curves, and triangles show results for the selected exoplanets following the same notation as Figure 9 . Cyan circles and diamonds show the observed (Lainey et al. 2017; Durante et al. 2020) and calculated (Wahl et al. 2017a, 2020) static $k_{22}$ for Jupiter and Saturn for comparison. Also labeled are the four exoplanet models with the largest nonlinearities.

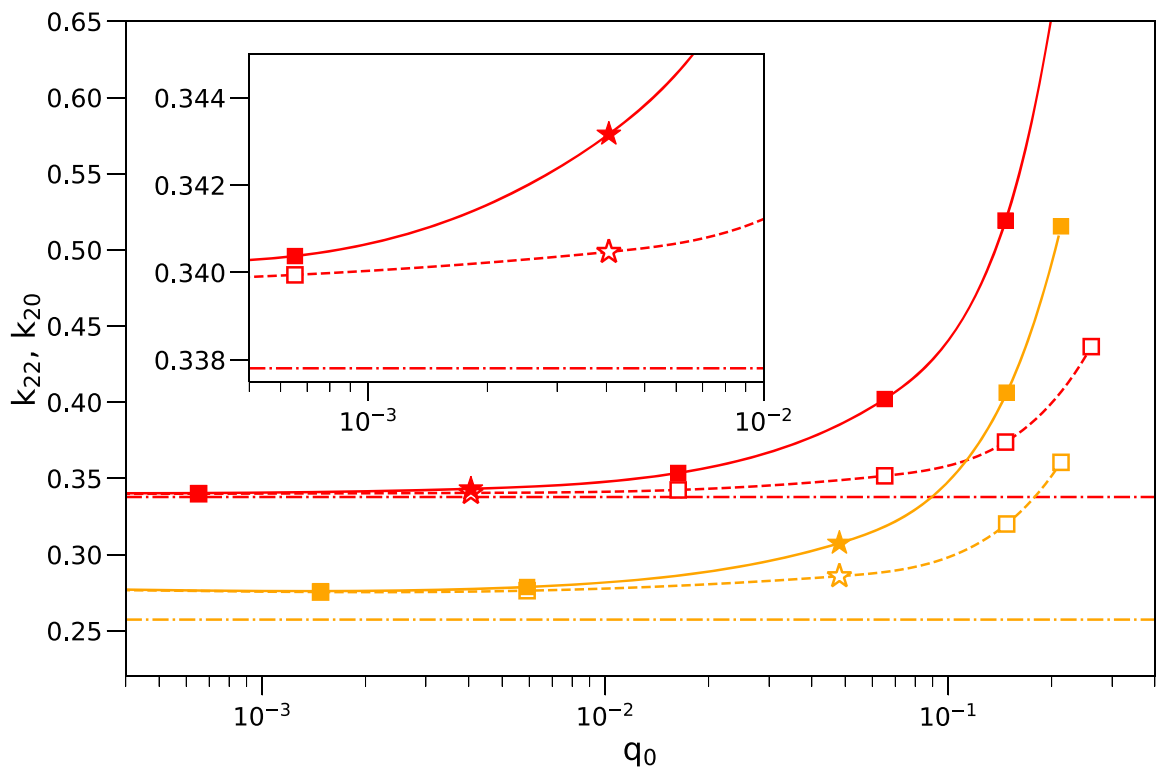

Figure 12. Variation of second-order Love numbers $k_{22}$ and $k_{20}$ with magnitude of the rotational parameter $q_{0}$. Red curves are for HAT-P-13b, and yellow are for WASP$121 \mathrm{~b}$. Here $k_{22}$ is a solid curve with filled squares, $k_{20}$ is dashed with open squares, and $k_{2 m}$ for the tidally locked state with the observed orbital period is denoted with stars. For each planet, the linear regime tidal response of $3 J_{2} / q_{0}$ is shown with a dashed-dotted line. Simulations for this figure use fewer $\left(N_{l}=128\right)$ CMS layers.

roughly 2 orders of magnitude larger than the tidally locked state. Figure 13 considers the complimentary exercise of raising the magnitude of $q_{\text {tid }}$ while maintaining $q_{0}$. In this case, the degree of splitting of $k_{22}$ and $k_{20}$ remains similar, while the overall magnitude increases for large magnitudes of $q_{\text {tid. }}$. For the two exoplanets considered here, both $q_{0}$ and $q_{\text {tid }}$ exhibit a comparable effect on the deviation from the linear regime response, with nonlinear response accounting for $\sim 16 \%$ of WASP-121b's $k_{22}$, but only $\sim 2 \%$ for HAT-P-13b.

When a tidally locked planet transits, the star's flux is dimmed by a disk of radius $\sqrt{b c}$. Leconte et al. (2011) compared this value to the radius of a nonrotating, unperturbed planet, $a_{0}$, and introduced the radius correction factor,

$$
\Delta R=\frac{a_{0}-\sqrt{b c}}{\sqrt{b c}}
$$

For WASP-12b, the largest correction in their data set, they obtained a $\Delta R$ range of $0.025-0.035$. When we use the same planet parameters, we find reasonable agreement with this correction, despite the fact that Leconte et al. (2011) based their work on the theory of figures, while we use the nonperturbative CMS theory here. They represented the interiors of hot Jupiters with a polytropic EOS, while we used a more realistic EOS for 


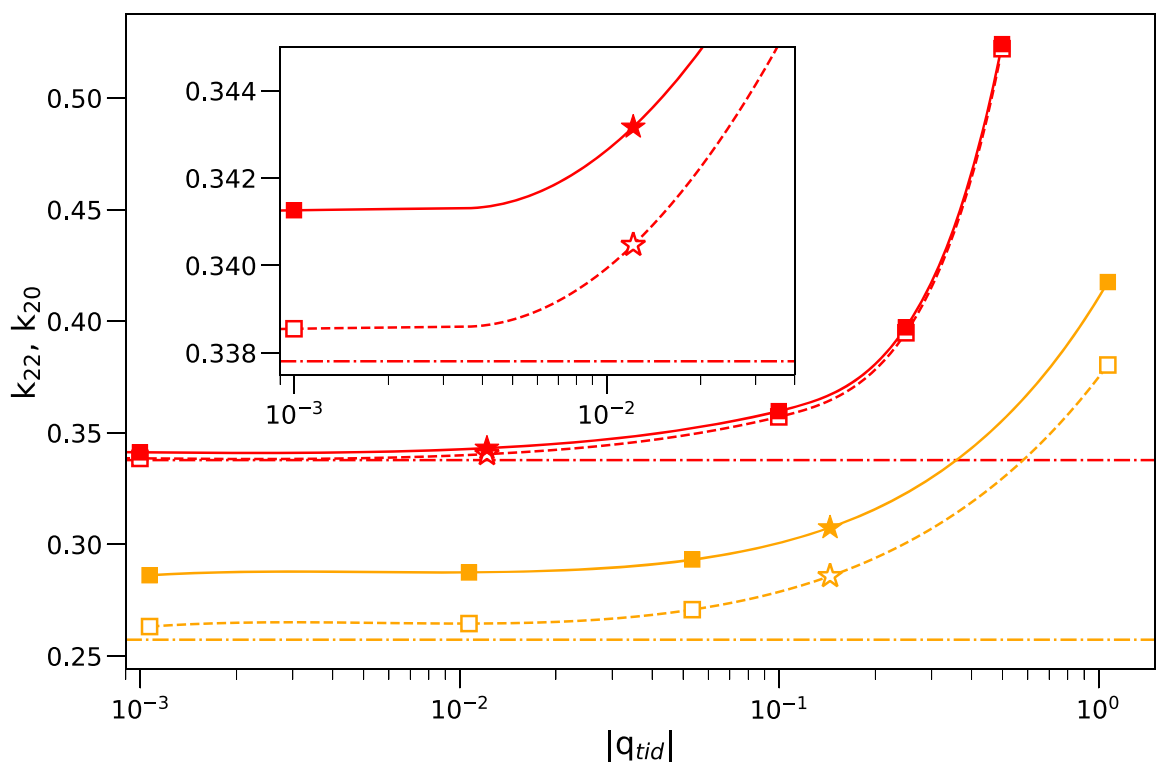

Figure 13. Variation of second-order Love numbers $k_{22}$ and $k_{20}$ with magnitude of the tidal parameter $q_{\text {tid. }}$ Quantities are depicted the same as in Figure 12 . Simulations for this figure use fewer $\left(N_{l}=128\right)$ CMS layers.

hydrogen-helium- $Z$ mixtures that was derived from ab initio computer simulations and yielded models for Jupiter and Saturn that agreed well with spacecraft observations (Wahl et al. 2017b; Iess et al. 2018). When we calculated $\Delta R$ for WASP-12b for $M=1.465 M_{\mathrm{J}}$ and $a_{0}=1.937 R_{\mathrm{J}}$, we obtained $\Delta R=0.0340$ for models with $M_{c}=0.293 M_{\mathrm{J}}$ and $Z=0$ and $\Delta R=0.0481$ for $M_{c}=0$ and $Z=0.19$. However, when we adopt the earlier parameters for WASP-12b, $M=1.404 M_{\mathrm{J}}$ and $a_{0}=1.736 R_{\mathrm{J}}$, we respectively obtain $\Delta R=0.0220$ and 0.0330 for our models with and without a core, a difference of only $\sim 6 \%$ from Leconte et al. (2011). In general, one finds that giant planets with large cores respond less to tidal perturbations, and thus their transit radius correction is smaller.

Akinsanmi et al. (2019) investigated how many transit observations with current instruments would be needed to determine the Love number of WASP-103b. They adopted a very wide range of shape Love number, $h_{22}$, ranging from 0.0 to 2.5 (for fluid planets, $h_{22}=1+k_{22}$ holds). Based on our interior models, we predict a much narrower range for this planet, with $k_{22}$ values between 0.109 and 0.388 , depending on whether the planet has a core of $0.588 M_{\mathrm{J}}$ or no core at all.

Correia (2014) assumed $k_{22}=0.5$ and studied the shape and light curves for a number of exoplanets. Hellard et al. (2019) investigated how $k_{22}$ can be derived from the transit light curves and constructed models for the shape of exoplanets under very specific assumptions. Qualitatively, our results for WASP-4b, WASP-12b, and WASP-18b in Table 3 agree with these model predictions, but one also notices some deviations. Correia (2014) predicted much larger values for the flattenings $f_{a c}$ and $f_{b c}$ of WASP-18b because they used a larger planet radius of $1.52 R_{\mathrm{J}}$, while we used $1.191 R_{\mathrm{J}}$. We derived a $k_{22}$ range from 0.226 to 0.424 rather than setting it to 0.5 . Our predictions for WASP- $18 \mathrm{~b}$ agree fairly well with Hellard et al. (2019), with only a small deviation for $f_{a c}$; they derived 0.0077 , which is slightly outside of our range of $0.0064-0.0074$.

For WASP-4b, the predictions by Hellard et al. (2019) for $f_{a b}, f_{b c}$, and $f_{a c}$ are all found to be slightly outside the range that is spanned by our models with and without cores. For example, we determined $f_{a c}=0.036-0.043$, while they predicted 0.045 .

For WASP-12b, we find better agreement with the shapes predicted by Correia (2014) once we adopt the older planet radius. However, even in this case, their $f_{b c}=0.031$ and that of Hellard et al. (2019), 0.036, are above our predicted range of 0.023-0.026 by $\sim 20 \%$.

Hellard et al. (2020) reported a tentative measurement of the WASP-121b Love number, $h_{22}=1.39_{-0.81}^{+0.71}$, which is compatible with the range of $h_{22}-1=k_{22}=0.122$ to 0.296 but not well constrained given the large reported uncertainty.

It is worth noting that in all cases summarized above, once parameters were selected to best match the previous estimates, our models consistently predict values of $f_{a c}, f_{b c}$, and $\Delta R$ that are slightly below the reported values. This suggests a systematic overestimation of the flattening by these models, possibly resulting from a less realistic hydrogen-helium EOS, neglecting nonlinear effects, or artificially constraining the surface shape to a perfect ellipsoid.

\section{Conclusions}

We studied the tidal response and shape of hot Jupiters, identified a number of general trends, and modeled eight specific exoplanets. Most tidally locked exoplanets are slowly rotating. They are thus in the linear regime, for which the Love number is well approximated by $k_{22}=3 J_{2} / q_{0}$ and cannot be greater than 0.6 . This limit was derived under realistic assumptions for giant-planet interiors in which the density strongly varies throughout the envelope. For close-in hot Jupiters, we studied how the high interior temperatures reduce the density profile of the envelope and demonstrate that this change further reduces $k_{22}$.

We also studied how the tidal response changes with increasing rotation rate. For extremely close-in hot Jupiters, we find that, in spite of tidal locking, rotation rates are sufficient to have a noticeable effect on the tidal response. For three of the selected exoplanets, WASP-12b, WASP-103b, and WASP-121b, we predict $k_{22}$ to exceed the linear value of $k_{22}=3 J_{2} / q_{0}$ by over $10 \%$, with the largest deviation of $\sim 19 \%$ for WASP-12. 
For realistic planet and stellar parameters, we find $k_{22}<0.45$ for all eight selected exoplanets. This limit is not compatible with much larger $k_{22}$ values that have been reported in the literature of a number of hot Jupiters (Hardy et al. 2017; Bouma et al. 2019; Csizmadia et al. 2019), which may indicate a systematic overestimation of $k_{22}$ by these observation methods. Only the observation of HAT-P-13b by Buhler et al. (2016) overlaps with our predicted $k_{22}$ range.

If the larger $k_{22}$ observations are confirmed, they imply either that these planets are fast-rotating and thus not tidally locked, or that dynamic tidal effects increase $k_{22}$ in ways that are not understood. For Jupiter, however, dynamic contributions to the tidal response (Idini \& Stevenson 2021; Lai 2021) have been shown to reduce the static value (Wahl et al. 2020) by approximately $\Delta k_{22} / k_{22}^{\text {static }} \approx 4 \%$, bringing it into agreement with observations made by the Juno spacecraft (Durante et al. 2020). For dynamic tidal effects to explain the discrepancy between the large observed values and our model predictions, they would not only have to have the opposite sign as for Jupiter but also be much larger in magnitude.

We compare our predictions for the shape of selected planets with earlier models by Leconte et al. (2011), Correia (2014), Akinsanmi et al. (2019), and Hellard et al. (2019) that relied on perturbative approaches and simpler assumptions for planetary interiors. Although we find reasonably good agreement for the shape and transit radius correction if we assume the same masses and radii as the other authors, we also suggest that these models may have a small but systematic overestimation of the planet's flattening.

This work was in part supported by the NASA mission Juno. B.M. acknowledges support from the Center for Matter at Atomic Pressure (CMAP) that is funded by the U.S. National Science Foundation (PHY-2020249). D.T. acknowledges support by the Trottier Fellowship from the Exoplanet Research Institute (iREx). This research has made use of the NASA Exoplanet Archive and data provided by the WASP consortium.

\section{ORCID iDs}

Sean M. Wahl (i) https://orcid.org/0000-0003-2451-7939 Daniel Thorngren (1) https://orcid.org/0000-0002-5113-8558 Tiger Lu (i) https://orcid.org/0000-0003-0834-8645 Burkhard Militzer (i) https://orcid.org/0000-0002-7092-5629

\section{References}

Akinsanmi, B., Barros, S. C., Santos, N. C., et al. 2019, A\&A, 621, A117 Batygin, K., Bodenheimer, P., \& Laughlin, G. 2009, ApJ, 704, 49 Becker, A., Bethkenhagen, M., Kellermann, C., Wicht, J., \& Redmer, R. 2018, AJ, 156,149

Becker, A., Lorenzen, W., Fortney, J. J., et al. 2015, ApJS, 215, 21 Bonomo, A. S., Sozzetti, A., Santerne, A., et al. 2015, A\&A, 575, A85 Bouma, L. G., Winn, J. N., Baxter, C., et al. 2019, AJ, 157, 217 Buhler, P., Knutson, H., Batygin, K., et al. 2016, ApJ, 821, 26 Burton, J. R., Watson, C. A., Fitzsimmons, A., et al. 2014, ApJ, 789, 113 Campo, C. J., Harrington, J., Hardy, R. A., et al. 2011, ApJ, 727, 125 Chabrier, G., Mazevet, S., \& Soubiran, F. 2019, ApJ, 872, 51

Chakrabarty, A., \& Sengupta, S. 2019, AJ, 158, 39

Charbonneau, D., Brown, T. M., Latham, D. W., \& Mayor, M. 2000, ApJL, 529, L45

Correia, A. C. 2014, A\&A, 570, L5

Correia, A. C. M., \& Rodríguez, A. 2013, ApJ, 767, 128

Csizmadia, S., Hellard, H., \& Smith, A. M. 2019, A\&A, 623, A45

Debras, F., \& Chabrier, G. 2019, ApJ, 872, 100

Deleuil, M., Deeg, H. J., Alonso, R., et al. 2008, A\&A, 491, 889
Delrez, L., Santerne, A., Almenara, J. M., et al. 2016, MNRAS, 458, 4025 Demory, B.-O., \& Seager, S. 2011, ApJS, 197, 12

Durante, D., Parisi, M., Serra, D., et al. 2020, GeoRL, 47, e86572

Folkner, W. M., Iess, L., Anderson, J. D., et al. 2017, GeoRL, 44, 4694

Fortney, J. J., Glenzer, S. H., Koenig, M., et al. 2009, PhPl, 16, 041003

Fortney, J. J., Marley, M. S., \& Barnes, J. W. 2007, ApJ, 659, 1661

Gavrilov, S. V., \& Zharkov, V. N. 1977, Icar, 32, 443

Gillon, M., Anderson, D. R., Collier-Cameron, A., et al. 2014, A\&A, 562, L3

Guillot, T., \& Showman, A. P. 2002, A\&A, 385, 156

H, A. R., \& Jeans, J. H. 1920, Geogr J., 56, 69

Hardy, R. A., Harrington, J., Hardin, M. R., et al. 2017, ApJ, 836, 143

Hellard, H., Csizmadia, S., Padovan, S., Sohl, F., \& Rauer, H. 2020, ApJ, 889,66

Hellard, H., Csizmadia, S., Spohn, T., \& Breuer, D. 2019, ApJ, 878, 119

Helled, R., Mazzola, G., \& Redmer, R. 2020, NatRP, 2, 562

Henry, G. W., Marcy, G. W., Butler, R. P., \& Vogt, S. S. 2000, ApJL, 529, L41

Hubbard, W. B. 2013, ApJ, 768, 43

Hubbard, W. B., \& Militzer, B. 2016, ApJ, 820, 80

Idini, B., \& Stevenson, D. J. 2021, PSJ, 2, 69

Iess, L., Folkner, W. M., Durante, D., et al. 2018, Natur, 555, 220

Iess, L., Militzer, B., Kaspi, Y., et al. 2019, Sci, 364, aat2965

Jackson, B., Greenberg, R., \& Barnes, R. 2008, ApJ, 678, 1396

Kellermann, C., Becker, A., \& Redmer, R. 2018, A\&A, 615, A39

Kramm, U., Nettelmann, N., Fortney, J. J., Neuhäuser, R., \& Redmer, R. 2012, A\&A, 538, A146

Lai, D. 2021, PSJ, 2, 122

Lainey, V., Casajus, L. G., Fuller, J., et al. 2020, NatAs, 4, 1053

Lainey, V., Jacobson, R. A., Tajeddine, R., et al. 2017, Icar, 281, 286

Leconte, J., Lai, D., \& Chabrier, G. 2011, A\&A, 528, A41

Lin, D. N., \& Gu, P. G. 2004, in First Astrophysics meeting of the Observatorio Astronomico Nacional, Gravitaional Collapse: From Massive Stars to Planets, 22, ed. G. García-Segura et al., 95

Lodders, K. 2010, in Formation and Evolution of Exoplanets, ed. R. Barnes (New York: Wiley), 157

Love, A. E. H. 1909, RSPSA, 82, 73

Mankovich, C. R., \& Fortney, J. J. 2020, ApJ, 889, 51

Mankovich, C. R., \& Fuller, J. 2021, NatAs

Miguel, Y., Guillot, T., \& Fayon, L. 2016, A\&A, 596, A114

Militzer, B., \& Hubbard, W. B. 2013, ApJ, 774, 148

Militzer, B., Soubiran, F., Wahl, S. M., \& Hubbard, W. 2016, JGRE, 121, 1552

Militzer, B., Wahl, S., \& Hubbard, W. 2019, ApJ, 879, 78

Miller, N., \& Fortney, J. J. 2011, ApJL, 736, L29

Mollière, P., van Boekel, R., Dullemond, C., Henning, T., \& Mordasini, C. 2015, ApJ, 813, 47

Morales, M. A., Pierleoni, C., Schwegler, E., \& Ceperley, D. M. 2010, PNAS, 107, 12799

NASA Exoplanet Science Institute 2011, NASA Exoplanet Archive, https:// exoplanetarchive.ipac.caltech.edu/cgi-bin/TblView/nph-tblView? $\mathrm{app}=$ ExoTbls\&config $=$ planets

Nettelmann, N. 2019, ApJ, 874, 156

Padovan, S., Spohn, T., Baumeister, P., et al. 2018, A\&A, 620, A178

Ragozzine, D., \& Wolf, A. S. 2009, ApJ, 698, 1778

Sarkis, P., Mordasini, C., Henning, T., Marleau, G. D., \& Mollière, P. 2021, A\&A, 645, A79

Saumon, D., Chabrier, G., \& van Horn, H. M. V. 1995, ApJS, 99, 713

Saumon, D., \& Guillot, T. 2004, ApJ, 609, 1170

Seager, S., \& Hui, L. 2002, ApJ, 574, 1004

Seager, S., Kuchner, M., Hier-Majumder, C. A., \& Militzer, B. 2007, ApJ, 669,1279

Shporer, A., Wong, I., Huang, C. X., et al. 2019, AJ, 157, 178

Thorngren, D., Gao, P., \& Fortney, J. J. 2019, ApJL, 884, L6

Thorngren, D. P., \& Fortney, J. J. 2018, AJ, 155, 214

Vorberger, J., Gericke, D. O., Bornath, T., \& Schlanges, M. 2010, PhRvE, 81, 046404

Wahl, S. M., Hubbard, W. B., \& Militzer, B. 2016, ApJ, 831, 14

Wahl, S. M., Hubbard, W. B., \& Militzer, B. 2017a, Icar, 282, 183

Wahl, S. M., Hubbard, W. B., Militzer, B., et al. 2017b, GeoRL, 44, 4649

Wahl, S. M., Parisi, M., Folkner, W. M., Hubbard, W. B., \& Militzer, B. 2020, ApJ, 891, 42

Wilson, H. F., \& Militzer, B. 2014, ApJ, 973, 34

Winn, J. N., Johnson, J. A., Howard, A. W., et al. 2010, ApJ, 718, 575

Zharkov, V. N. 2004, AstL, 30, 496

Zharkov, V. N., \& Gudkova, T. V. 2010, P\&SS, 58, 1381

Zharkov, V. N., \& Trubitsyn, V. P. 1978, in The Physics of Planetary Interiors, ed. W. B. Hubbard (Tucson, AZ: Parchart), 380 